\title{
CONVERGENCE RESULTS OF THE FICTITIOUS DOMAIN METHOD FOR A MIXED FORMULATION OF THE WAVE EQUATION WITH A NEUMANN BOUNDARY CONDITION*
}

\author{
Eliane Bécache ${ }^{1}$, Jeronimo Rodríguez ${ }^{2}$ And Chrysoula Tsogka ${ }^{3}$
}

\begin{abstract}
The problem of modeling acoustic waves scattered by an object with Neumann boundary condition is considered. The boundary condition is taken into account by means of the fictitious domain method, yielding a first order in time mixed variational formulation for the problem. The resulting system is discretized with two families of mixed finite elements that are compatible with mass lumping. We present numerical results illustrating that the Neumann boundary condition on the object is not always correctly taken into account when the first family of mixed finite elements is used. We, therefore, introduce the second family of mixed finite elements for which a theoretical convergence analysis is presented and error estimates are obtained. A numerical study of the convergence is also considered for a particular object geometry which shows that our theoretical error estimates are optimal.
\end{abstract}

Mathematics Subject Classification. 65M60, 65M12, 65M15, 65C20, 74S05.

Received February 22, 2008. Revised September 22, 2008.

Published online December 5, 2008.

\section{INTRODUCTION}

This work falls within a more general framework of developing efficient numerical methods for approximating wave propagation in complex media such as anisotropic, heterogeneous media with cracks or objects of arbitrary shapes. We consider here the scattering of acoustic waves by perfect reflectors, i.e., objects or cracks with a homogeneous Neumann boundary condition. To solve these wave propagation problems in an efficient way we use a fictitious domain approach. This approach, also called the domain embedding method, consists in extending artificially the solution to the interior of the object so that the new domain of computation has a very simple shape (typically a rectangle in $2 \mathrm{D}$ ). To account for the boundary condition, a new auxiliary unknown, defined only on the boundary of the object, is introduced. The solution of this extended problem has now a singularity across the boundary of the object which can be related to the new unknown. The main advantage of the method is that the mesh for the solution on the enlarged domain can be chosen independently

Keywords and phrases. Mixed finite elements, fictitious domain method, domain embedding method, acoustic waves, convergence analysis.

* The work of C. Tsogka was partially supported by the European FP7 Marie Curie International Reintegration Grant MIRGCT-2007-203438.

1 POEMS, INRIA-Rocquencourt, BP 105, 78153 Le Chesnay Cédex, France. eliane.becache@inria.fr

2 POEMS, ENSTA, 32 boulevard Victor, 75739 Paris Cedex 15, France. jeronimo.rodriguez@ensta.fr

3 Dept. of Applied Mathematics, University of Crete \& IACM/FORTH, Crete, Greece. tsogka@tem.uoc.gr 
of the geometry of the object. In particular, one can use regular grids or structured meshes which allows for simple and efficient computations.

Special interest has been given to this approach as it has been shown to lead to efficient numerical methods for a large number of applications (e.g. $[1,14,16-18,20,21,24])$ and recently for time dependent wave propagation problems $[4,6,12,13,23,26]$. The method can be re-interpreted in terms of a minimization problem in which case the auxiliary unknown appears as a Lagrange multiplier associated to the boundary condition viewed now as an equality constraint in the functional space. Thus the key point of the approach is that it can be applied to essential type boundary conditions, i.e., conditions that can be considered as equality constraints.

To do so with the free surface condition, the dual unknown (which is the velocity $\mathbf{v}$ here) has to be one of the unknowns. This can be done by considering either the dual formulation (the formulation with only one unknown, the dual one) or the mixed dual primal formulation. In both cases, the dual unknown is introduced and sought for in the space $H$ (div) in which the Neumann boundary condition $\mathbf{v} \cdot n$ can be considered as an equality constraint. In this case, the Lagrange multiplier is simply the jump of the primal unknown (the pressure $p$ ) across the boundary of the object.

For the approximation of the mixed formulation in the scalar acoustic case, in [3], the authors have proposed mixed finite elements, the so-called $Q_{k+1}^{\text {div }}-Q_{k}$ elements, inspired by Nédélec's second family [25]. These elements are compatible with mass lumping, and therefore allow for constructing explicit schemes in time. The generalization of those elements to the case of elastic waves was introduced in [2] for the stress-velocity formulation.

A non standard convergence analysis of the $Q_{k+1}^{\text {div }}-Q_{k}$ elements has been carried out in [3] for their scalar version and in [5] for their elastodynamic vectorial version. However this convergence analysis only concerned the velocity-pressure (resp. stress-velocity) mixed problem without an obstacle, that is, it did not address the convergence of the fictitious domain method.

In [4], the authors considered the scattering of elastic waves by a crack. The tools for solving this problem were the fictitious domain method (for modeling the crack) combined with $Q_{1}^{\text {div }}-Q_{0}$ elements (for the discretization of the volumetric unknowns). The numerical illustrations, done with a straight horizontal crack, seemed to show the convergence of the method. However, in [8], it was shown considering other configurations of cracks (e.g. diagonal cracks) that this approach did not always lead to a convergent method. Motivated by this negative result, we introduced a modified finite element, the so-called $Q_{1}^{\text {div }}-P_{1}^{\text {disc }}$, that numerically ensures convergence. However, in the elastodynamic case the theoretical convergence is still an open question.

In this paper the convergence of the fictitious domain method is analyzed for the scalar problem. Section 1 presents the fictitious domain method and its approximation. In Section 2, we address the question of its convergence when using $Q_{1}^{\text {div }}-Q_{0}$ elements for approximating the volumetric unknowns and it is shown in Section 2.2 through numerical experiments that the method does not always converge. In Section 3 we introduce the scalar version of the modified finite element $Q_{1}^{\text {div }}-P_{1}^{\text {disc }}$. We illustrate with numerical results the convergence for this modified element in Section 3.2. Due to the enrichment of the approximation space for the pressure field we observe the introduction of spurious modes in the solution. To get rid of this non physical part of the solution we propose to attenuate these spurious modes in Section 3.3 by introducing a damping term in the equations. Section 4 is devoted to the convergence analysis of the fictitious domain method when using the $Q_{1}^{\text {div }}-P_{1}^{\text {disc }}$ element. The theoretical order of convergence is compared to the numerical one in Section 5 for a particular object, comparison which confirms the optimality of the theoretical estimates.

\section{Fictitious domain Formulation of the DifFraCtion PROBlem}

\subsection{The continuous problem}

Let $C \subset \mathbb{R}^{2}$ be a bounded domain of "simple" geometry (typically a rectangle) with boundary $\Sigma$ and $\Gamma \subset C$ be a smooth curve without self-intersections. All of what follows can be applied to both a closed curve or an open curve $\Gamma$, but for the sake of clarity we will consider the latter. We assume that $\Gamma$ can be extended to a closed smooth curve $\widetilde{\Gamma} \subset C$ dividing the domain $C=\Omega_{i} \cup \widetilde{\Gamma} \cup \Omega_{e}$ into two sub-domains $\Omega_{i}$ and $\Omega_{e}$ (see Fig. 1(b)). 


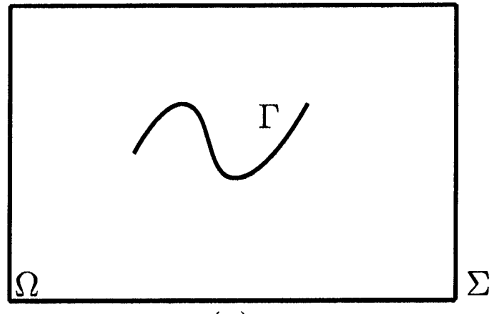

(a)

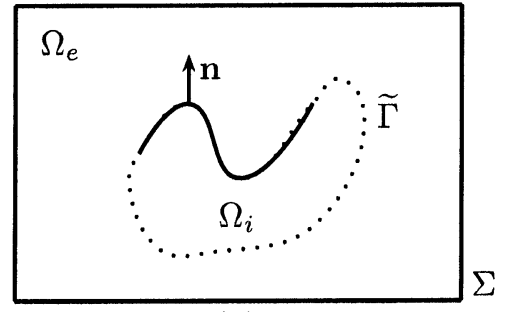

(b)

FiguRE 1. Geometry of the problem.

In this case, $\widetilde{\Gamma}=\partial \Omega_{i}$ and $\Sigma \cup \widetilde{\Gamma}=\partial \Omega_{e}$. We consider the propagation of acoustic waves on the domain $\Omega=C \backslash \Gamma$ when the pressure field is subject to a homogeneous Neumann boundary condition on the boundary $\Gamma$. The propagation medium is assumed to be anisotropic and the equation satisfied by the pressure field is the scalar wave equation. In order to apply the fictitious domain method to this type of boundary condition it is customary (e.g. [3]) to formulate the problem as a first-order velocity-pressure system,

$$
\left\{\begin{array}{rlr}
\text { Find }(\mathbf{v}, p):(x, t) & \in \times[0, T] \mapsto(\mathbf{v}(x, t), p(x, t)) \in \mathbb{R}^{2} \times \mathbb{R} \text { satisfying, } \\
\rho \frac{\partial p}{\partial t}-\operatorname{div} \mathbf{v} & =f, \quad \text { in } \Omega, \\
\mathbf{A} \frac{\partial \mathbf{v}}{\partial t}-\nabla p & =0, \quad \text { in } \Omega, \\
\mathbf{v} \cdot n & =0, \quad \text { on } \Gamma, \\
p & =0, \quad \text { on } \Sigma,
\end{array}\right.
$$

with the initial conditions,

$$
p(t=0)=p_{0} ; \mathbf{v}(t=0)=\mathbf{v}_{0},
$$

where the unknowns $p$ and $\mathbf{v}$ denote the pressure and the velocity field. The scalar function $\rho$ and the tensor $\mathbf{A}$ characterize the propagation medium and $f$ represents the external forces. Moreover, we assume that $\rho$ satisfies

$$
0<\rho^{-} \leq \rho(x) \leq \rho^{+}<+\infty
$$

and $\mathbf{A}$ is a second order symmetric positive tensor such that

$$
0<\kappa|\mathbf{w}|^{2} \leq \mathbf{A}(x) \mathbf{w} \cdot \mathbf{w} \leq \nu|\mathbf{w}|^{2}, \quad \forall \mathbf{w} \neq 0
$$

We also assume that the support of the initial data $\left(\mathbf{v}_{0}, p_{0}\right)$ and the support of the source $f$ do not intersect $\Gamma$, which means that

$$
\operatorname{supp}\left(\mathbf{v}_{0}\right) \cup \operatorname{supp}\left(p_{0}\right) \subset C \backslash \Gamma, \quad \bigcup_{t \leq T} \operatorname{supp}(f(t)) \subset C \backslash \Gamma .
$$


The natural variational formulation of this problem would be set in some functional spaces that depend on the shape of the obstacle (i.e., depend on $\Omega$ ). More precisely, the classical variational formulation is,

$$
\left\{\begin{array}{rlrl}
\text { Find }(\mathbf{v}(t), p(t)) \in \underline{X}_{0} \times M \text { satisfying, } & \\
\frac{\mathrm{d}}{\mathrm{d} t} \int_{\Omega} \mathbf{A v} \cdot \mathbf{w} \mathrm{d} x+\int_{\Omega} \operatorname{div}(\mathbf{w}) p \mathrm{~d} x & =0, & & \forall \mathbf{w} \in \underline{X}_{0}, \\
\frac{\mathrm{d}}{\mathrm{d} t} \int_{\Omega} \rho p q \mathrm{~d} x-\int_{\Omega} \operatorname{div}(\mathbf{v}) q \mathrm{~d} x & =(f, q), & \forall q \in M, \\
(\mathbf{v}, p)_{/ t=0} & =\left(\mathbf{v}_{0}, p_{0}\right), &
\end{array}\right.
$$

where the functional spaces are defined as,

$$
\underline{X}_{0}(\Omega)=\{\mathbf{w} \in H(\operatorname{div} ; \Omega), \mathbf{w} \cdot \mathbf{n}=0, \text { on } \Gamma\}, M=L^{2}(\Omega) .
$$

Remark 1.1. In the definition of $\underline{X}_{0}(\Omega)$ the normal trace $\mathbf{w} \cdot \mathbf{n}$ is defined by duality as follows. For any function $\mu \in H_{00}^{1 / 2}(\Gamma)$ one can define its extension by zero $\tilde{\mu} \in H^{1 / 2}(\widetilde{\Gamma})$, where $\widetilde{\Gamma}$ is a closed extension of $\Gamma$ (see Sect. 1.1 and Fig. $1(\mathrm{~b})$ ). We will denote by $\Omega_{i}$ (resp. $\Omega_{e}$ ) the interior (resp. exterior) domain to $\widetilde{\Gamma}$. There exists $q_{i} \in H^{1}\left(\Omega_{i}\right)$ such that $q_{i}=\tilde{\mu}$ on $\widetilde{\Gamma}$. We now define $(\mathbf{w} \cdot \mathbf{n})_{\Gamma}^{i}$ by duality as the element of $\left(H_{00}^{1 / 2}(\Gamma)\right)^{\prime}$ such that

$$
\left\langle(\mathbf{w} \cdot \mathbf{n})_{\Gamma}^{i}, \mu\right\rangle_{\left(H_{00}^{1 / 2}(\Gamma)\right)^{\prime}, H_{00}^{1 / 2}(\Gamma)}=\int_{\Omega_{i}} \operatorname{div} \mathbf{w} q_{i} \mathrm{~d} x+\int_{\Omega_{i}} \mathbf{w} \cdot \nabla q_{i} \mathrm{~d} x .
$$

It can be proven that the right hand side of (1.5) does not depend on the lifting $q_{i}$ but only on $\mu$ and that it defines a unique element of $\left(H_{00}^{1 / 2}(\Gamma)\right)^{\prime}$. We will define $H^{-1 / 2}(\Gamma):=\left(H_{00}^{1 / 2}(\Gamma)\right)^{\prime}$. We can define in the same way the exterior normal trace $(\mathbf{w} \cdot \mathbf{n})_{\Gamma}^{e}$. For an element $\mathbf{w} \in \underline{X}_{0}(\Omega)$ both traces coincide and are equal to zero.

We also introduce the norm on $H_{00}^{1 / 2}(\Gamma)$ as

$$
\|\mu\|_{H_{00}^{\frac{1}{2}}(\Gamma)}=\|\tilde{\mu}\|_{H^{\frac{1}{2}}(\widetilde{\Gamma})}=\inf _{q \in H^{1}(C) / q_{\mid \widetilde{\Gamma}}=\tilde{\mu}}\left(\int_{C} q^{2} \mathrm{~d} x+\int_{C}|\nabla q|^{2} \mathrm{~d} x\right)^{1 / 2}
$$

We can establish the following result concerning the well posedness of problem (1.4).

Theorem 1.1. Let $f \in C^{1}([0, T], M), \mathbf{v}_{0} \in \underline{X}_{0}(\Omega), p_{0} \in H^{1}(\Omega)$ satisfy (1.3). Then, problem (1.4) has a unique solution $(\mathbf{v}, p)$ such that

$$
\begin{aligned}
& \mathbf{v} \in C^{1}\left([0, T],\left(L^{2}(\Omega)\right)^{2}\right) \cap C^{0}\left([0, T], \underline{X}_{0}(\Omega)\right), \\
& p \quad \in \quad C^{1}([0, T], M) \cap C^{0}\left([0, T], H^{1}(\Omega)\right) .
\end{aligned}
$$

Proof. Under the assumptions of the theorem one can easily show the existence and uniqueness of a strong solution for problem (1.1) using the Hille-Yoshida theorem [22]. This solution will also satisfy (1.4). The uniqueness is obtained by energy arguments.

The fictitious domain formulation of this problem consists in taking into account the boundary condition on $\Gamma$ in a weak way, by introducing a Lagrange multiplier $\lambda$ defined on $\Gamma$. This allows for working in functional spaces (for the volume unknowns) which do not depend any more on the shape of the obstacle. The fictitious domain formulation is then the following (to simplify the notations, we will still denote by $(\mathbf{v}(t), p(t))$ the new 
unknowns defined now in $C$ )

$$
\left\{\begin{array}{rlrl}
\text { Find }(\mathbf{v}(t), p(t), \lambda(t)) \in \underline{X} \times M \times \mathcal{G} \text { satisfying, } & \\
\frac{\mathrm{d}}{\mathrm{d} t} a(\mathbf{v}, \mathbf{w})+b(\mathbf{w}, p)-\langle\mathbf{w} \cdot \mathbf{n}, \lambda\rangle_{\Gamma} & =0, & & \forall \mathbf{w} \in \underline{X}, \\
\frac{\mathrm{d}}{\mathrm{d} t}(p, q)_{\rho}-b(\mathbf{v}, q) & =(f, q), & & \forall q \in M, \\
& \langle\mathbf{v} \cdot \mathbf{n}, \mu\rangle_{\Gamma} & =0, & \\
(\mathbf{v}, p)_{/ t=0} & & =\left(\mathbf{v}_{0}, p_{0}\right), &
\end{array}\right.
$$

where the functional spaces are now defined as,

$$
\underline{X}(=\underline{X}(C))=H(\operatorname{div} ; C), M=L^{2}(C), \mathcal{G}=H_{00}^{1 / 2}(\Gamma),
$$

the bilinear forms as,

$$
\left\{\begin{aligned}
a(\mathbf{v}, \mathbf{w})=\int_{C} \mathbf{A} \mathbf{v} \cdot \mathbf{w} \mathrm{d} x, & \forall(\mathbf{v}, \mathbf{w}) \in \underline{X} \times \underline{X}, \\
(p, q)_{\eta}=\int_{C} \eta p q \mathrm{~d} x, & \forall(p, q) \in M \times M, \\
b(\mathbf{w}, q)=\int_{C} \operatorname{div}(\mathbf{w}) q \mathrm{~d} x, & \forall(\mathbf{w}, q) \in \underline{X} \times M,
\end{aligned}\right.
$$

and the bracket $\langle\mathbf{w} \cdot \mathbf{n}, \mu\rangle_{\Gamma}$ is the duality product between $\mathcal{G}$ and $\mathcal{G}^{\prime}$. Note that, due to (1.3), under assumptions of Theorem 1.1, the data also belong to

$$
f \in C^{0}([0, T], M), \quad \mathbf{v}_{0} \in \underline{X}(C), \quad p_{0} \in M .
$$

In the following we will denote by $\|q\|_{\eta}:=(q, q)_{\eta}^{1 / 2}$ and $(\cdot, \cdot):=(\cdot, \cdot)_{1}$ the usual $L^{2}(C)$ scalar product. We also introduce the usual norms on $\underline{X}$ and $M$ by:

$$
\|p\|_{M}:=\|p\|, \quad\|\mathbf{v}\|_{\underline{X}}:=\left(\int_{C}|\mathbf{v}|^{2} \mathrm{~d} x+\int_{C}|\operatorname{div} \mathbf{v}|^{2} \mathrm{~d} x\right)^{1 / 2} .
$$

The well posedness of problem (1.7) follows from the three following lemmas.

Lemma 1.1 (existence). We assume the data $\left(\mathbf{v}_{0}, p_{0}, f\right)$ satisfy $(1.9)$. Let $(\mathbf{v}, p) \in\left(C^{1}\left([0, T],\left(L^{2}(\Omega)\right)^{2}\right) \cap\right.$ $\left.C^{0}\left([0, T], \underline{X}_{0}(\Omega)\right)\right) \times C^{1}([0, T], M)$ be the solution of problem (1.4). Then:

(i) $p \in C^{0}\left([0, T], H^{1}(\Omega)\right)$ and one can define

$$
\lambda=[p]_{\Gamma} \in C^{0}([0, T], \mathcal{G}),
$$

where $[p]_{\Gamma}$ denotes the jump of $p$ across $\Gamma$.

(ii) $\mathbf{v} \in C^{0}([0, T], \underline{X}(C))$ and $(\mathbf{v}, p, \lambda)$ is a solution of $(1.7)$.

Proof. (i) If $(\mathbf{v}, p) \in\left(C^{1}\left([0, T],\left(L^{2}(\Omega)\right)^{2}\right) \cap C^{0}\left([0, T], \underline{X}_{0}(\Omega)\right)\right) \times C^{1}([0, T], M)$ is the solution of (1.4), the reinterpretation of the variational formulation shows that it satisfies in particular (1.1)-(b) in $\left(L^{2}(\Omega)\right)^{2}$. Since $\partial_{t} v \in C^{0}\left([0, T],\left(L^{2}(\Omega)\right)^{2}\right)$ we deduce that $\nabla p \in C^{0}\left([0, T],\left(L^{2}(\Omega)\right)^{2}\right)$ and therefore $p \in C^{0}\left([0, T], H^{1}(\Omega)\right)$. It is then possible to define its trace on $\Gamma$ and define $\lambda$.

(ii) $\mathbf{v}$ is in $C^{0}\left([0, T], \underline{X}_{0}(\Omega)\right)$ and by the definition of $\underline{X}_{0}(\Omega)$ the normal trace of the velocity field $\mathbf{v} \cdot \mathbf{n}$ on both sides of the crack coincide (is equal to zero), thus $\mathbf{v} \in C^{0}([0, T], \underline{X}(C))$. Again using the re-interpretation of 
the variational formulation, one can see that (1.1)-(a) is satisfied in $L^{2}(\Omega),(1.1)-(\mathrm{b})$ in $\left(L^{2}(\Omega)\right)^{2}$ and $(1.1)$-(d) in $H^{1 / 2}(\Sigma)$. We then easily check that it satisfies (1.7), by multiplying (1.1)-(a) with a function $q \in M,(1.1)$-(b) with a function $\mathbf{w} \in \underline{X}$, integrating by parts and using the definition of $\lambda$.

Lemma 1.2 (energy identity). If $(\mathbf{v}, p, \lambda)$ is a solution of $(1.7)$, the energy

$$
\mathcal{E}=\frac{1}{2} a(\mathbf{v}, \mathbf{v})+\frac{1}{2}\|p\|_{\rho}^{2}
$$

satisfies the following identity,

$$
\frac{\mathrm{d} \mathcal{E}}{\mathrm{d} t}=(f, p)
$$

Lemma 1.3. The following inf-sup condition is satisfied,

$$
\exists k>0, \forall \mu \in \mathcal{G}, \exists \mathbf{w} \in \underline{X},\langle\mathbf{w} \cdot \mathbf{n}, \mu\rangle_{\Gamma} \geq k\|\mu\|_{\mathcal{G}}\|\mathbf{w}\|_{\underline{X}} .
$$

Proof. This result has been proved in [23] for a closed obstacle $\widetilde{\Gamma}$, and the corresponding space $\widetilde{\mathcal{G}}=H^{1 / 2}(\widetilde{\Gamma})$. It is straightforward to adapt the proof to the present case, extending the open curve $\Gamma$ to a closed curve $\widetilde{\Gamma}$, since for any function $\mu \in H_{00}^{1 / 2}(\Gamma)$ one can define its extension by zero $\widetilde{\mu} \in H^{1 / 2}(\widetilde{\Gamma})$. We then apply the result for $\widetilde{\mu}$ and using $\|\widetilde{\mu}\|_{\tilde{\mathcal{G}}}=\|\mu\|_{\mathcal{G}}$ and $\langle\mathbf{w} \cdot \mathbf{n}, \tilde{\mu}\rangle_{\widetilde{\Gamma}}=\langle\mathbf{w} \cdot \mathbf{n}, \mu\rangle_{\Gamma}$ we obtain the result for $\mu$.

Theorem 1.2. Let $f \in C^{0}([0, T], M), \mathbf{v}_{0} \in \underline{X}, p_{0} \in M$ satisfying (1.3). Problem (1.7) admits a unique solution $(\mathbf{v}, p, \lambda) \in\left(C^{1}\left([0, T],\left(L^{2}(C)\right)^{2}\right) \cap C^{0}([0, T], \underline{X})\right) \times C^{1}([0, T], M) \times C^{0}([0, T], \mathcal{G})$.

Proof. The existence follows from Lemma 1.1. The energy identity (1.10) implies the uniqueness of (v, $p$ ) and the uniqueness of $\lambda$ is a consequence of the inf-sup condition (1.11).

Remark 1.2. On the regularity of the solution. For smooth enough data, one can expect more regularity on the solution. However, in general, the space regularity of the volumetric part of the solution is at most,

$$
\mathbf{v}(t) \in H^{\frac{1}{2}-\varepsilon}(\operatorname{div}, C), \quad p(t) \in H^{\frac{1}{2}-\varepsilon}(C), \quad \varepsilon>0
$$

and this is obtained for sufficiently smooth data and sufficiently smooth geometries. This is due to the fact that the unknowns are defined on the whole domain $C$ without considering the geometry of the obstacle.

The regularity in $\Omega$ (i.e. outside the obstacle) is in general higher and depends on the geometry of the obstacle. In particular, for data $\left(\mathbf{v}_{0}, p_{0}, f\right)$ satisfying $(1.9)$, we have

- for a closed smooth boundary:

$$
p_{/ \Omega}(t) \in H^{2}(\Omega), \quad \lambda(t) \in H^{3 / 2}(\Gamma)
$$

- for an open boundary, due to the singular behavior near the tip of the crack (the solution behaves as $\sqrt{r}, r$ being here the distance to the tip; see Chapters 2 and 5 of [19] and references therein for further details), we have

$$
p_{/ \Omega}(t) \in H^{3 / 2-\varepsilon}(\Omega), \quad \lambda(t) \in H^{1-\varepsilon}(\Gamma), \quad \varepsilon>0 .
$$




\subsection{The semi-discrete approximation}

For the spatial approximation of this problem, we introduce finite dimensional spaces $\underline{X_{h}} \subset \underline{X}, M_{h} \subset M$ and $\mathcal{G}_{H} \subset \mathcal{G}$ satisfying the approximation properties,

$$
\begin{cases}\lim _{h \rightarrow 0} \inf _{h}\left\|\underline{X_{h}}\right\| \mathbf{v}-\mathbf{w}_{h} \|_{\underline{X}}=0, \quad \forall \mathbf{v} \in \underline{X}, \\ \lim _{h \rightarrow 0} \inf _{q_{h} \in M_{h}}\left\|p-q_{h}\right\|_{M}=0, \quad \forall p \in M \\ \lim _{H \rightarrow 0} \inf _{\mu_{H} \in \mathcal{G}_{H}}\left\|\lambda-\mu_{H}\right\|_{\mathcal{G}}=0, & \forall \lambda \in \mathcal{G} .\end{cases}
$$

The semi-discrete problem is then,

$$
\left\{\begin{array}{lrll}
\text { Find }\left(\mathbf{v}_{h}(t), p_{h}(t), \lambda_{H}(t)\right) \in \underline{X_{h}} \times M_{h} \times \mathcal{G}_{H} \text { such that, } & \\
\frac{\mathrm{d}}{\mathrm{d} t} a\left(\mathbf{v}_{h}, \mathbf{w}_{h}\right)+b\left(\mathbf{w}_{h}, p_{h}\right)-\left\langle\mathbf{w}_{h} \cdot \mathbf{n}, \lambda_{H}\right\rangle_{\Gamma}=0, & \forall \mathbf{w}_{h} \in \underline{X_{h}}, \\
\frac{\mathrm{d}}{\mathrm{d} t}\left(p_{h}, q_{h}\right)_{\rho}-b\left(\mathbf{v}_{h}, q_{h}\right) & \left\langle\mathbf{v}_{h} \cdot \mathbf{n}, \mu_{H}\right\rangle_{\Gamma}=0, & \left.\forall q_{h}\right), & \forall q_{h} \in M_{h}, \\
& & & \\
\mathbf{v}_{h}(t=0) & =\mathbf{v}_{h, 0}, & & \\
p_{h}(t=0) & =p_{h, 0}, &
\end{array}\right.
$$

where $\left(\mathbf{v}_{h, 0}, p_{h, 0}\right) \in X_{h} \times M_{h}$ is an approximation of the exact initial condition.

The question is: How to choose the approximate spaces in order to ensure the convergence of $\left(\mathbf{v}_{h}, p_{h}, \lambda_{H}\right)$ to $(\mathbf{v}, p, \lambda)$ ?

\section{The fictitious domain method using the $Q_{1}^{\text {div }}-Q_{0}$ ELEment}

\subsection{Formulation of the problem}

For the volumetric unknowns, we introduce a regular mesh $\mathcal{T}_{h}$ of the rectangular domain $C$ composed of square elements of length $h$. In [3], we introduced for the problem without obstacle new mixed finite elements, the so-called $Q_{k+1}^{\text {div }}-Q_{k}$ elements, inspired by Nédélec's second family [25]. The choice of these elements is related to the fact that we are concerned with time domain propagation problems and we want to use explicit schemes in time. The finite elements have then to be compatible with mass lumping, which means that the mass matrices are approximated using quadrature formulas by diagonal (or block diagonal) matrices. To do so, the quadrature nodes have to be localized at the same place as the degrees of freedom. This can be achieved with the second family of Nedelec's elements (see [3] for more details). It is well known [25] that these elements do not enter the classical theory of mixed finite elements [10,15] (lack of coercivity, see below). However, a non standard convergence analysis of these $Q_{k+1}^{\text {div }}-Q_{k}$ elements has been carried out, showing the convergence without the fictitious domain method. Our first choice for the approximation spaces of the problem with an obstacle was naturally the lowest order element $Q_{1}^{\text {div }}-Q_{0}$ for the velocity and the pressure fields,

$$
\left\{\begin{array}{l}
\underline{X_{h}}=\left\{\mathbf{w}_{h} \in \underline{X} / \forall K \in \mathcal{T}_{h},\left.\mathbf{w}_{h}\right|_{K} \in Q_{1} \times Q_{1}\right\}, \\
M_{h}=M_{h}^{0} \text { with } \quad M_{h}^{0}=\left\{q_{h} \in M / \forall K \in \mathcal{T}_{h},\left.q_{h}\right|_{K} \in Q_{0}\right\} .
\end{array}\right.
$$

The degrees of freedom for the mixed element are described in Figure 2. For more details on this element we refer to [3]. Notice that the velocity approximation space $\underline{X_{h}}$ contains the lower order Raviart Thomas element,

$$
{\underline{X_{h}}}^{R T}=\left\{\mathbf{w}_{h} \in \underline{X} / \forall K \in \mathcal{T}_{h},\left.\mathbf{w}_{h}\right|_{K} \in P_{10} \times P_{01}\right\} .
$$




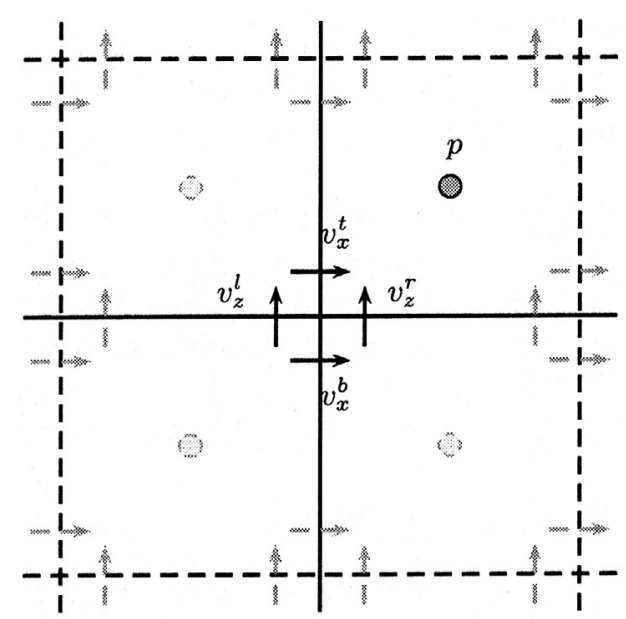

Figure 2. Degrees of freedom for the $Q_{1}^{\text {div }} \times Q_{0}$ mixed finite element.

For the approximation of the Lagrange multiplier, we introduce a mesh of $\Gamma$ composed of $N$ segments $S_{j}$ of length $H_{j}$, and we set $H=\sup _{j} H_{j}$. We assume that this mesh is quasiuniform:

$$
\exists \nu, 0<\nu \leq 1 \text {, such that: } \forall j, 1 \leq j \leq N, H_{j} \geq \nu H .
$$

We then choose the space of continuous linear piecewise functions:

$$
\mathcal{G}_{H}=\left\{\nu_{H} \in \mathcal{G} / \forall S_{j}, j=1, \ldots, N,\left.\nu_{H}\right|_{S_{j}} \in P_{1}\right\} .
$$

The spaces $\left(\underline{X_{h}}, M_{h}^{0}, \mathcal{G}_{H}\right)$ clearly satisfy the approximation properties in (1.12).

This choice of spaces seemed to be natural since the finite element given by $\left(\underline{X_{h}}, M_{h}^{0}\right)$ converges in the absence of an obstacle [3] and the space $\mathcal{G}_{H}$ provides one of the simplest conforming approximation of $\mathcal{G}$. However we have not been able to prove the convergence of the fictitious domain method with these spaces. The convergence analysis of the fictitious domain method applied to other problems $[1,14,23]$ shows that convergence holds if a compatibility condition between the step sizes of the two meshes is satisfied,

$$
H \geq \alpha h .
$$

We will show in what follows some numerical illustrations which seem to indicate that for some special configurations of obstacles, the method does not converge.

Before showing these numerical results, let us briefly recall the main difficulty of the convergence analysis in the case without object. Introducing the linear operators,

$$
\begin{aligned}
& B: \frac{X}{\mathbf{w}} \longrightarrow \begin{array}{c}
M^{\prime} \\
\mapsto B(\mathbf{w}): M \longrightarrow \mathbb{R}
\end{array} \\
& q \mapsto\langle B(\mathbf{w}), q\rangle=b(\mathbf{w}, q),
\end{aligned}
$$

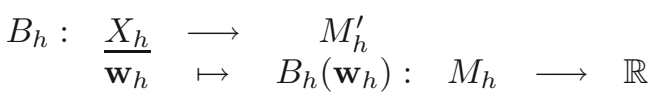

$$
\begin{aligned}
& q_{h} \mapsto\left\langle B_{h}\left(\mathbf{w}_{h}\right), q_{h}\right\rangle=b\left(\mathbf{w}_{h}, q_{h}\right),
\end{aligned}
$$

it is easy to verify that the bilinear form $a(.,$.$) is not coercive on \operatorname{Ker}\left(B_{h}\right)$ (even though it is on $\operatorname{Ker}(B)$, but we do not have $\operatorname{Ker}\left(B_{h}\right) \subset \operatorname{Ker}(B)$ ), so that our problem does not fit the classical mixed finite element theory 
(cf. $[10,15])$. It was however possible to overcome this difficulty when dealing with the problem without coupling with the fictitious domain method. When coupled with the fictitious domain method, the same technique cannot be applied.

\subsection{Numerical illustrations}

The computational domain is the square $[0,10] \mathrm{mm} \times[0,10] \mathrm{mm}$ composed of a homogeneous isotropic material with $\rho=1000 \mathrm{Kg} \cdot \mathrm{m}^{-3}$ and $\mathbf{A}=I \times 10^{9} \mathrm{~Pa}$. It is excited by an initial condition on the pressure centered at $\left(x_{c}, z_{c}\right)=(5,5) \mathrm{mm}$

$$
p((x, z), t=0)=0.1 F\left(\frac{r}{r_{0}}\right)
$$

where $F(r)$ is supported in $[0,1]$ and given by (for $r \in[0,1]$ )

$$
F(r)=A_{0}-A_{1} \cos (2 \pi r)+A_{2} \cos (3 \pi r)-A_{3} \cos (6 \pi r),
$$

with $\mathbf{r}=\left(x-x_{c}, z-z_{c}\right)^{t}, r=\|\mathbf{r}\|, r_{0}=1 \mathrm{~mm}$ and

$$
A_{0}=0.35875, \quad A_{1}=0.48829, \quad A_{2}=0.14128, \quad A_{3}=0.01168 .
$$

We consider a uniform mesh of squares using a discretization step $h=0.025 \mathrm{~mm}$. The time discretization is done using a leap frog scheme with the time step $\Delta t$ chosen in such a way that the ratio $\Delta t / h$ is equal to the maximal value that ensures the stability. Perfectly matched layers are used to simulate a non bounded domain on all the boundaries.

Horizontal obstacle. In the first experiment we consider a plane horizontal crack

$$
(x, z)=(5+2 \sqrt{2}(2 t-1), 5-2 \sqrt{2}) \mathrm{mm}, \quad t \in[0,1],
$$

that we discretize using a uniform mesh of step $H=R h$. The method seems to converge and we obtain good results for reasonable values of the parameter $R$ (in the interval $[0.75,3]$ ). In particular, the wave is well reflected by the crack as expected. In the first column of Figure 3 we show a snapshot of the pressure field for $R=1.2$.

Diagonal obstacle. In the second experiment we treat a plane diagonal defect given by

$$
(x, z)=(5+4 t, 1+4 t) \mathrm{mm}, \quad t \in[0,1],
$$

that is, the same obstacle considered in the previous paragraph rotated by $\pi / 4$ radians with respect to $\left(x_{c}, z_{c}\right)$, the center of the initial condition. As the medium is isotropic, the solution of the continuous problem is also a rotation of the solution when considering the horizontal crack.

We discretize the Lagrange multiplier using again a uniform mesh of step $H=R h$ with several values for the parameter $R$. However, this time, the approximate solution does not seem to converge towards the physical solution. In particular, the incident wave is not completely reflected but also transmitted through the crack (see for instance the second panel of Fig. 3 for $R=1.2$ ). Other numerical examples show the same phenomenon; even after refining the mesh, the amplitude of the transmitted wave does not diminish. There is no convergence with a diagonal obstacle.

\section{The modified Element $Q_{1}^{\text {div }}-P_{1}^{\text {disc }}$}

\subsection{Presentation of the modified element}

In Section 2.1, we have conjectured that the difficulty of the convergence analysis comes from the lack of coercivity of $a(\cdot, \cdot)$ on $\operatorname{Ker}\left(B_{h}\right)$. In order to overcome this problem, we propose to modify the space $M_{h}$ in such 


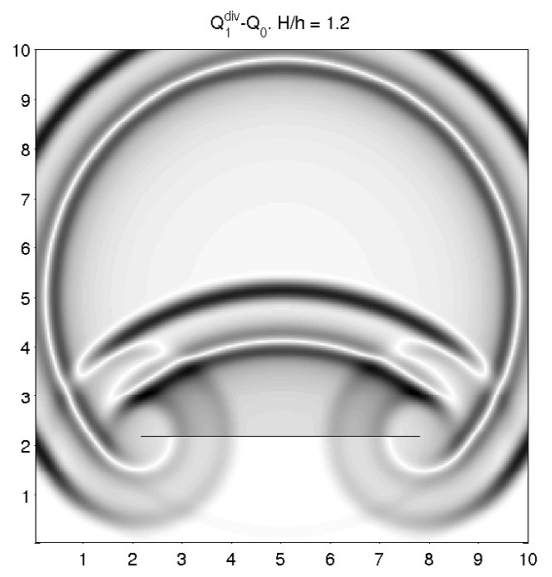

(a) Horizontal defect
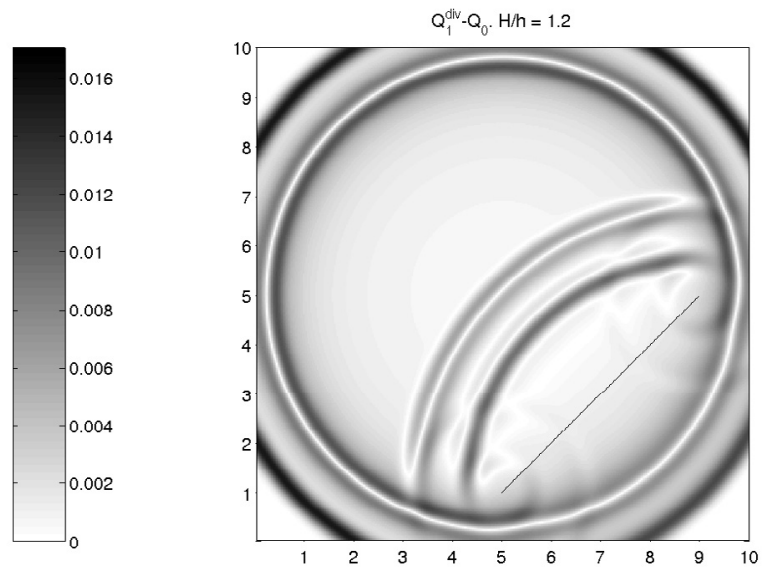

(b) Diagonal defect

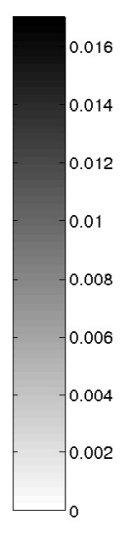

Figure 3. Snapshots of the pressure field at time $t=5.3033012 \mu$ s obtained with the $Q_{1}^{\text {div }}-Q_{0}$ finite element method for $H / h=1.2$.

a way that

$$
\operatorname{div}\left(\underline{X_{h}}\right) \subset M_{h}
$$

which implies

$$
\operatorname{Ker}\left(B_{h}\right) \subset \operatorname{Ker}(B)
$$

providing the coercivity of $a(\cdot, \cdot)$ on $\operatorname{Ker}\left(B_{h}\right)$. This might simplify the analysis. That is why we have chosen to discretize the pressure in the space

$$
M_{h}=M_{h}^{1} \quad \text { with } \quad M_{h}^{1}=\left\{q_{h} \in M / \forall K \in \mathcal{T}_{h},\left.q_{h}\right|_{K} \in P_{1}(K)\right\}
$$

Consequently, we will have three degrees of freedom per element on the unknown $p_{h}$ as shown in Figure 4 . Since $M_{h}^{0} \subset M_{h}^{1}$ we have obviously

$$
\inf _{q_{h} \in M_{h}}\left\|p-q_{h}\right\|_{M} \leq \inf _{q_{h}^{0} \in M_{h}^{0}}\left\|p-q_{h}^{0}\right\|_{M}
$$

so that the approximation properties (1.12) are still satisfied.

Remark 3.1. Assuming (3.1) and that the density is constant on each element we have that

$$
\mathbf{w}_{h} \in \underline{X_{h}} \Longrightarrow q_{h}:=\frac{1}{\rho} \operatorname{div}\left(\mathbf{w}_{h}\right) \in M_{h}
$$

Introducing this particular test function in the second equation of (1.13) we obtain

$$
\frac{\mathrm{d}}{\mathrm{d} t} \int_{C} p_{h} \operatorname{div}\left(\mathbf{w}_{h}\right) \mathrm{d} x-\int_{C} \frac{1}{\rho} \operatorname{div}\left(\mathbf{v}_{h}\right) \operatorname{div}\left(\mathbf{w}_{h}\right) \mathrm{d} x=\int_{C} \frac{1}{\rho} f \operatorname{div}\left(\mathbf{w}_{h}\right) \mathrm{d} x .
$$




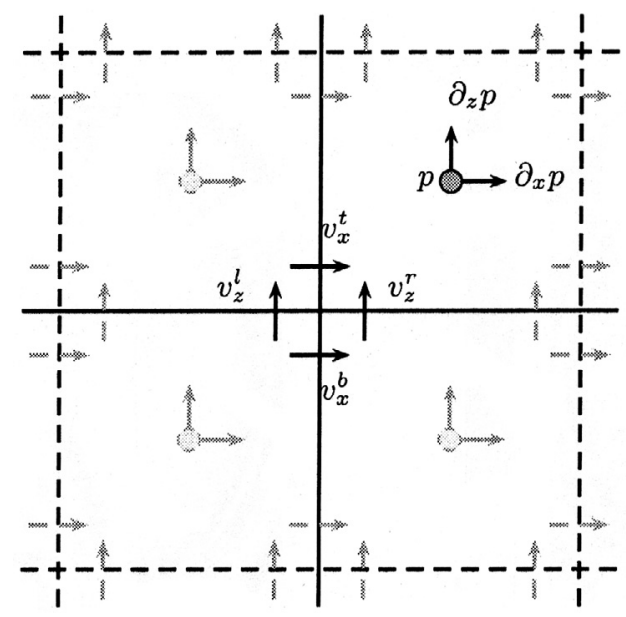

Figure 4. Degrees of freedom for the $Q_{1}^{\text {div }} \times P_{1}^{\text {disc }}$ mixed finite element.

Deriving with respect to time the first and third equations of (1.13) and using the last expression we deduce that our variational formulation implies the following second order formulation

$$
\left\{\begin{array}{l}
\text { Find }\left(\mathbf{v}_{h}(t), \tilde{\lambda}_{H}(t)\right) \in \underline{X_{h}} \times \mathcal{G}_{H} \text { such that } \forall\left(\mathbf{w}_{h}, \mu_{H}\right) \in \underline{X}_{h} \times \mathcal{G}_{H} \\
\frac{\mathrm{d}^{2}}{\mathrm{~d} t^{2}} \int_{C} \mathbf{A} \mathbf{v}_{h} \cdot \mathbf{w}_{h} \mathrm{~d} x+\int_{C} \frac{1}{\rho} \operatorname{div}\left(\mathbf{w}_{h}\right) \operatorname{div}\left(\mathbf{v}_{h}\right) \mathrm{d} x-\int_{\Gamma} \mathbf{w}_{h} \cdot \mathbf{n} \tilde{\lambda}_{H} \mathrm{~d} \gamma=\int_{C}-\frac{1}{\rho} f \operatorname{div}\left(\mathbf{w}_{h}\right) \mathrm{d} x \\
\int_{\Gamma} \mathbf{v}_{h} \cdot \mathbf{n} \mu_{H} \mathrm{~d} \gamma=0 \\
\mathbf{v}_{h}(t=0)=\mathbf{v}_{h, 0}, \\
p_{h}(t=0)=p_{h, 0}
\end{array}\right.
$$

where $\tilde{\lambda}_{H}=\frac{\partial \lambda_{H}}{\partial t}$. The nature of this problem is close to those analyzed in [14,23].

\subsection{Some numerical illustrations of the fictitious domain method using the modified element}

Let us now show some numerical illustrations of the behavior of the fictitious domain method with the new finite element space. The numerical experiments that we have considered are the same as in Section 2.2 and will allow us to compare both finite elements.

Horizontal obstacle. Once again we discretize the horizontal crack defined by (2.5) using a uniform mesh of step $H=R h$. The results obtained with the new mixed finite element $Q_{1}^{\text {div }}-P_{1}^{\text {disc }}$ are similar to those given by the $Q_{1}^{\text {div }}-Q_{0}$ element. The method converges for reasonable values of the parameter $R$ (in the interval $[0.75,3])$. In the first column of Figure 5 we can see the results for $R=1.2$.

Diagonal obstacle. We now consider the diagonal crack defined by the expression (2.6). We recall that the continuous problem is a rotation of $\pi / 4$ radians with respect to the point $\left(x_{c}, z_{c}\right)=(5,5)$ of the one presented on the previous paragraph. The Lagrange multiplier is again discretized using a uniform mesh of step $H=R h$. Contrary to the results obtained with the element $Q_{1}^{\text {div }}-Q_{0}$, those given by the modified element $Q_{1}^{\text {div }}-P_{1}^{\text {disc }}$ seem to show the convergence of the numerical solution towards the physical solution when choosing reasonable values for the ratio $H / h$ (see the second column of Fig. 5 for $R=1.2$ ). This result will be proved in Section 4 . 


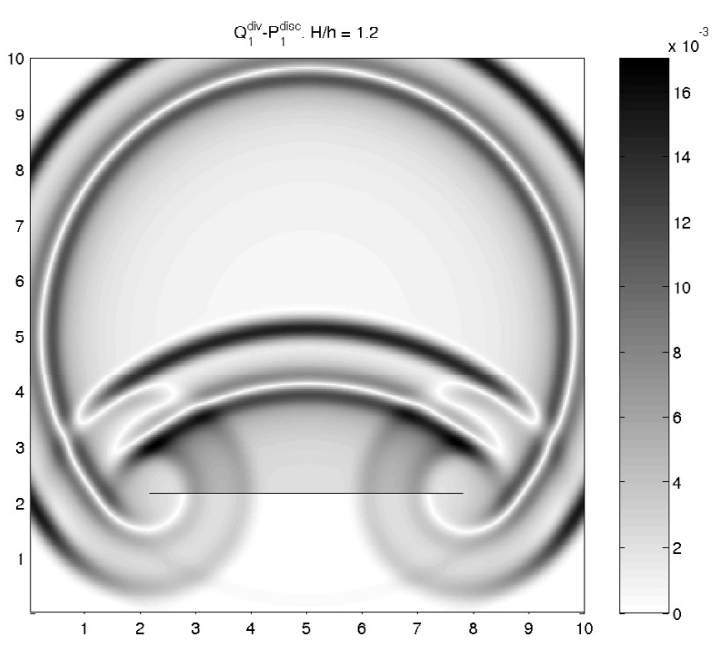

(a) Horizontal defect

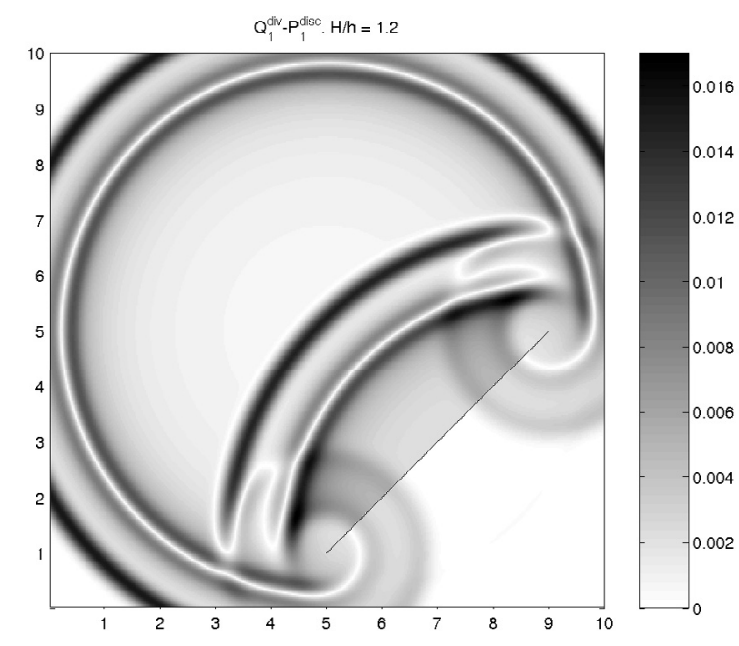

(b) Diagonal defect

Figure 5. Snapshots of the pressure field at time $t=5.3033012 \mu$ s obtained with the $Q_{1}^{\text {div }}-$ $P_{1}^{\text {disc }}$ finite element method for $H / h=1.2$.

\subsection{Presence of spurious modes and their damping}

Let us remark that the modified space $M_{h}^{1}$ can be decomposed as

$$
M_{h}=M_{h}^{0} \oplus M_{h}^{r}
$$

where $M_{h}^{0}$ is the space of piecewise constants and $M_{h}^{r}$ is its orthogonal complement (for the $L^{2}$ scalar product). The space $M_{h}^{r}$ is composed of $P_{1}$ discontinuous functions with vanishing mean value per element.

Due to this enrichment of the approximation space for the pressure field, spurious modes that contaminate the discrete solution appear when using the modified element. These spurious modes can be characterized further using a dispersion analysis and it is easy to prove $[7,8]$ that their main components belong to $M_{h}^{r}$. In order to damp this main part of the spurious modes, we introduce the following approximate problem (instead of (1.13)): find $\left(p_{h}, \mathbf{v}_{h}\right) \in M_{h} \times \underline{X_{h}}$ such that

$$
\left\{\begin{array}{l}
\frac{\mathrm{d}}{\mathrm{d} t} a\left(\mathbf{v}_{h}, \mathbf{w}_{h}\right)+b\left(\mathbf{w}_{h}, p_{h}\right)-\left\langle\mathbf{w}_{h} \cdot n, \lambda_{H}\right\rangle_{\Gamma}=0, \quad \forall \mathbf{w}_{h} \in \underline{X_{h}}, \\
\frac{\mathrm{d}}{\mathrm{d} t}\left(p_{h}, q_{h}\right)_{\rho}+\left(P_{M_{h}^{r}}\left(p_{h}\right), q_{h}\right)_{\beta}-b\left(\mathbf{v}_{h}, q_{h}\right)=\left(f, q_{h}\right), \quad \forall q_{h} \in M_{h}, \\
\left\langle\mathbf{v}_{h} \cdot n, \mu_{H}\right\rangle_{\Gamma}=0, \quad \forall \mu_{H} \in \mathcal{G}_{H},
\end{array}\right.
$$

where for any subspace $\widetilde{M}_{h} \subset M_{h}, P_{\widetilde{M}_{h}}(\cdot)$ denotes the $L^{2}$ orthogonal projection of $M_{h}$ on $\widetilde{M}_{h}$, defined for any $\varphi_{h} \in M_{h}$ as,

$$
P_{\widetilde{M}_{h}}\left(\varphi_{h}\right) \in \widetilde{M}_{h} \quad \text { and } \quad\left(P_{\widetilde{M}_{h}}\left(\varphi_{h}\right), q_{h}\right)=\left(\varphi_{h}, q_{h}\right), \quad \forall q_{h} \in \widetilde{M}_{h}
$$

To simplify the notation we also set

$$
\varphi_{h}^{r}=P_{M_{h}^{r}}\left(\varphi_{h}\right), \quad \varphi_{h}^{0}=P_{M_{h}^{0}}\left(\varphi_{h}\right), \quad \forall \varphi_{h} \in M_{h}
$$


In system (3.5) $\beta$ represents a damping parameter, which is chosen as a positive constant in practice. The case $\beta=0$ gives back the non-damped problem (i.e., system (1.13)), while a strictly positive $\beta$ corresponds to a dissipative problem. From the numerical point of view, it remains to define a procedure in order to choose this parameter in an appropriate way (see [7] for an empirical way of choosing that parameter).

\section{Convergence analysis}

In this section we show the convergence of the fictitious domain method using the modified element with damping. The proof of convergence is composed of two main steps. One step consists in relating, using energy techniques, error estimates for the evolution problem to terms involving the difference between the exact solution and its elliptic projection (that has to be cleverly defined). The second step amounts to analyzing the elliptic projection error and we will start with this point.

\subsection{Elliptic projection error}

We define the elliptic projection operator in the following way:

$$
(\mathbf{v}, p, \lambda) \in \underline{X} \times M \times \mathcal{G} \rightarrow \Pi_{h}(\mathbf{v}, p, \lambda)=\left(\widehat{\mathbf{v}}_{h}, \widehat{p}_{h}, \widehat{\lambda}_{H}\right) \in \underline{X_{h}} \times M_{h} \times \mathcal{G}_{H},
$$

where $\left(\widehat{\mathbf{v}}_{h}, \widehat{p}_{h}, \widehat{\lambda}_{H}\right) \in \underline{X_{h}} \times M_{h} \times \mathcal{G}_{H}$ is solution of

$$
\left\{\begin{array}{l}
\left(\widehat{p}_{h}-p, q_{h}\right)-b\left(\widehat{\mathbf{v}}_{h}-\mathbf{v}, q_{h}\right)=0, \quad \forall q_{h} \in M_{h}, \\
a\left(\widehat{\mathbf{v}}_{h}-\mathbf{v}, \mathbf{w}_{h}\right)+b\left(\mathbf{w}_{h}, \widehat{p}_{h}-p\right)-\left\langle\mathbf{w}_{h} \cdot \mathbf{n}, \widehat{\lambda}_{H}-\lambda\right\rangle_{\Gamma}=0, \quad \forall \mathbf{w}_{h} \in \underline{X_{h}}, \\
\left\langle\left(\widehat{\mathbf{v}}_{h}-\mathbf{v}\right) \cdot \mathbf{n}, \mu_{H}\right\rangle_{\Gamma}=0, \quad \forall \mu_{H} \in \mathcal{G}_{H} .
\end{array}\right.
$$

It is easy to show that this problem is equivalent to defining first the couple $\left(\widehat{\mathbf{v}}_{h}, \widehat{\lambda}_{H}\right) \in \underline{X_{h}} \times \mathcal{G}_{H}$ satisfying

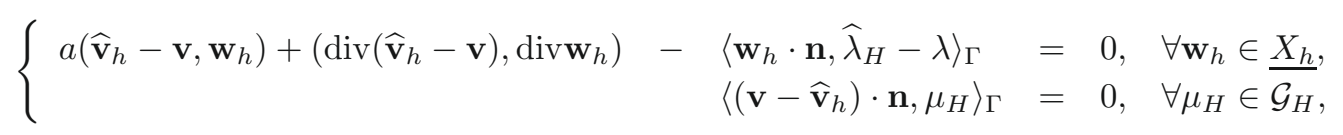

and then $\widehat{p}_{h} \in M_{h}$ satisfying

$$
\left(\widehat{p}_{h}-p, q_{h}\right)=b\left(\widehat{\mathbf{v}}_{h}-\mathbf{v}, q_{h}\right), \quad \forall q_{h} \in M_{h} .
$$

This follows from the fact that $\operatorname{div} \underline{X_{h}} \subset M_{h}$, so that we can choose $q_{h}=\operatorname{div} \mathbf{w}_{h}$. It is well known that the convergence of $\left(\widehat{\mathbf{v}}_{h}, \widehat{\lambda}_{H}\right)$ to $(\mathbf{v}, \lambda)$ is related to the uniform discrete inf-sup condition:

$$
\left\{\begin{array}{l}
\exists C>0 \text { independent of } h \text { such that } \\
\forall \mu_{H} \in \mathcal{G}_{H}, \exists \mathbf{w}_{h} \in \underline{X_{h}},\left\langle\mathbf{w}_{h} \cdot \mathbf{n}, \mu_{H}\right\rangle_{\Gamma} \geq C\left\|\mathbf{w}_{h}\right\|_{\underline{X}}\left\|\mu_{H}\right\|_{\mathcal{G}} .
\end{array}\right.
$$

Theorem 4.1. If assumption (2.2) is satisfied, then there exists a constant $\alpha>0$ such that if $H \geq \alpha$, the uniform discrete inf-sup condition (4.4) is satisfied for spaces $\left(\underline{X_{h}}, \mathcal{G}_{H}\right)$.

Proof. The result has been shown in [23] for the couple of spaces $\left(\underline{X}_{h}{ }^{R T}, \mathcal{G}_{H}\right)$. The space $\underline{X}_{h}$ considered here clearly contains $\underline{X}_{h}^{R T}$ (cf. [3]), which shows that the inf-sup condition is still true for the couple $\left(\underline{X_{h}}, \mathcal{G}_{H}\right)$.

Once the inf-sup condition is satisfied, there is no difficulty in applying the classical Babuška-Brezzi [10] theory and we obtain the elliptic projection estimates: 
Theorem 4.2. We assume that $H \geq \alpha$ h where $\alpha$ is the constant given in Theorem 4.1. The problem (4.1) has a unique solution $\left(\widehat{p}_{h}, \widehat{\mathbf{v}}_{h}, \widehat{\lambda}_{H}\right) \in M_{h} \times \underline{X_{h}} \times \mathcal{G}_{H}$ which satisfies

$$
\begin{aligned}
\left\|\mathbf{v}-\widehat{\mathbf{v}}_{h}\right\|_{\underline{X}}+\left\|\lambda-\widehat{\lambda}_{H}\right\|_{\mathcal{G}} & \leq C\left(\inf _{\mathbf{w}_{h} \in \underline{X_{h}}}\left\|\mathbf{v}-\mathbf{w}_{h}\right\|_{\underline{X}}+\inf _{\mu_{H} \in \mathcal{G}_{H}}\left\|\lambda-\mu_{H}\right\|_{\mathcal{G}}\right), \\
\left\|p-\widehat{p}_{h}\right\|_{M} & \leq C\left(\inf _{q h \in M_{h}}\left\|p-q_{h}\right\|_{M}+\inf _{\mathbf{w}_{h} \in \underline{X_{h}}}\left\|\mathbf{v}-\mathbf{w}_{h}\right\|_{\underline{X}}+\inf _{\mu_{H} \in \mathcal{G}_{H}}\left\|\lambda-\mu_{H}\right\|_{\mathcal{G}}\right) .
\end{aligned}
$$

Proof. The error estimates for $\left(\mathbf{v}-\widehat{\mathbf{v}}_{h}, \lambda-\widehat{\lambda}_{H}\right)$ follow from the classical theory [10]. For $\widehat{p}_{h}$, we use (4.3) which implies that

and then

$$
\left\|\widehat{p}_{h}-q_{h}\right\|_{M} \leq\left\|p-q_{h}\right\|_{M}+\left\|\operatorname{div}\left(\widehat{\mathbf{v}}_{h}-\mathbf{v}\right)\right\|, \quad \forall q_{h} \in M_{h},
$$

$$
\left\|\widehat{p}_{h}-p\right\|_{M} \leq 2 \inf _{q_{h} \in M_{h}}\left\|p-q_{h}\right\|_{M}+\left\|\widehat{\mathbf{v}}_{h}-\mathbf{v}\right\|_{\underline{X}} .
$$

In the following theorem we give a finer result. We show that the pressure is approximated with the piecewise constant part of the solution $p_{h}$ (projection on $M_{h}^{0}$ ) while the rest (projection on $M_{h}^{r}$ ) tends to zero.

Theorem 4.3. We assume that $H \geq \alpha$ where $\alpha$ is the constant given in Theorem 4.1. If $\left(\widehat{p}_{h}^{0}, \widehat{p}_{h}^{r}\right)=$ $\left(P_{M_{h}^{0}}\left(\widehat{p}_{h}\right), P_{M_{h}^{r}}\left(\widehat{p}_{h}\right)\right) \in M_{h}^{0} \times M_{h}^{r}$ denote the orthogonal projections of $\widehat{p}_{h}$ on $M_{h}^{0}$ and $M_{h}^{r}$, we have:

$$
\left\|\widehat{p}_{h}^{0}-p\right\|_{M}+\left\|\widehat{p}_{h}^{r}\right\|_{M} \leq C\left(\inf _{\mathbf{w}_{h} \in \underline{X_{h}}}\left\|\mathbf{v}-\mathbf{w}_{h}\right\|_{\underline{X}}+\inf _{\mu_{H} \in \mathcal{G}_{H}}\left\|\lambda-\mu_{H}\right\|_{\mathcal{G}}+\inf _{q_{h}^{0} \in M_{h}^{0}}\left\|p-q_{h}^{0}\right\|_{M}\right) .
$$

Proof. Using equation (4.3) for $q_{h}^{0} \in M_{h}^{0}$, all the terms in $M_{h}^{r}$ disappear,

$$
\left(\widehat{p}_{h}^{0}-p, q_{h}^{0}\right)=b\left(\widehat{\mathbf{v}}_{h}-\mathbf{v}, q_{h}^{0}\right), \quad \forall q_{h}^{0} .
$$

With the same arguments as previously we obtain,

$$
\left\|\widehat{p}_{h}^{0}-p\right\|_{M} \leq C\left(\left\|\operatorname{div}\left(\widehat{\mathbf{v}}_{h}-\mathbf{v}\right)\right\|+\inf _{q_{h}^{0} \in M_{h}^{0}}\left\|p-q_{h}^{0}\right\|_{M}\right) .
$$

Since $\widehat{p}_{h}^{r}=\widehat{p}_{h}-p+p-\widehat{p}_{h}^{0}$, it suffices to combine both estimates (4.6) and the first estimate of (4.7) to obtain the estimate on $\widehat{p}_{h}^{r}$.

Remark 4.1. The elliptic projection of time dependent functions $(\mathbf{v}, p, \lambda)$ depends also on time and it is easy to check that if

$$
(\mathbf{v}, p, \lambda) \in C^{k}([0, T] ; \underline{X} \times M \times \mathcal{G}),
$$

then

$$
\Pi_{h}(\mathbf{v}, p, \lambda) \in C^{k}\left([0, T] ; \underline{X}_{h} \times M_{h} \times \mathcal{G}_{H}\right),
$$

and

$$
\frac{\partial^{k}}{\partial t^{k}} \Pi_{h}(\mathbf{v}, p, \lambda)=\Pi_{h}\left(\frac{\partial^{k} \mathbf{v}}{\partial t^{k}}, \frac{\partial^{k} p}{\partial t^{k}}, \frac{\partial^{k} \lambda}{\partial t^{k}}\right) .
$$

We will also need in the following error estimates on the time derivative of the elliptic projection,

Corollary 4.1. We assume that $H \geq \alpha$ where $\alpha$ is the constant given in Theorem 4.1 and that $(\mathbf{v}, p, \lambda)$ depend on time $t$ and

$$
(\mathbf{v}, p, \lambda) \in \mathcal{C}^{k}([0, T] ; \underline{X} \times M \times \mathcal{G}) .
$$


Then

$$
\begin{aligned}
\left\|\partial_{t}^{k}\left(\widehat{\mathbf{v}}_{h}-\mathbf{v}\right)\right\|_{\underline{X}}+\left\|\partial_{t}^{k}\left(\widehat{\lambda}_{H}-\lambda\right)\right\|_{\mathcal{G}} \leq & \mathcal{C}\left(\inf _{\mathbf{w}_{h} \in \underline{X}_{h}}\left\|\mathbf{w}_{h}-\partial_{t}^{k} \mathbf{v}\right\|_{\underline{X}}+\inf _{\mu_{H} \in \mathcal{G}_{H}}\left\|\mu_{H}-\partial_{t}^{k} \lambda\right\|_{\mathcal{G}}\right), \\
\left\|\partial_{t}^{k}\left(\widehat{p}_{h}-p\right)\right\|_{M} \leq & \mathcal{C}\left(\inf _{\mathbf{w}_{h} \in \underline{X}_{h}}\left\|\mathbf{w}_{h}-\partial_{t}^{k} \mathbf{v}\right\|_{\underline{X}}\right. \\
& \left.+\inf _{q_{h} \in M_{h}}\left\|q_{h}-\partial_{t}^{k} p\right\|_{M}+\inf _{\mu_{H} \in \mathcal{G}_{H}}\left\|\mu_{H}-\partial_{t}^{k} \lambda\right\|_{\mathcal{G}}\right) \\
\left\|P_{M_{h}^{0}}\left(\partial_{t}^{k} \widehat{p}_{h}\right)-\partial_{t}^{k} p\right\|_{M}+\left\|P_{M_{h}^{r}}\left(\partial_{t}^{k} \widehat{p}_{h}\right)\right\|_{M} \leq & \mathcal{C}\left(\inf _{\mathbf{w}_{h} \in \underline{X}_{h}}\left\|\mathbf{w}_{h}-\partial_{t}^{k} \mathbf{v}\right\|_{\underline{X}}\right. \\
& \left.+\inf _{q_{h}^{0} \in M_{h}^{0}}\left\|q_{h}^{0}-\partial_{t}^{k} p\right\|_{M}+\inf _{\mu_{H} \in \mathcal{G}_{H}}\left\|\mu_{H}-\partial_{t}^{k} \lambda\right\|_{\mathcal{G}}\right) .
\end{aligned}
$$

Finally, assuming more regularity on the solution, classical approximation properties for finite elements give estimates with respect to $h$, i.e., for $\varepsilon>0$ :

$$
\left\{\begin{aligned}
\inf _{\mathbf{w}_{h} \in \underline{X_{h}}}\left\|\mathbf{v}-\mathbf{w}_{h}\right\|_{\underline{X}} \leq C h^{1 / 2-\varepsilon}\|\mathbf{v}\|_{H_{\text {div }}^{\frac{1}{2}-\varepsilon},}, & \forall \mathbf{v} \in H^{\frac{1}{2}-\varepsilon}(\operatorname{div}, C), \\
\inf _{q_{h} \in M_{h}}\left\|p-q_{h}\right\|_{M} \leq C h^{1 / 2-\varepsilon}\|p\|_{H^{\frac{1}{2}-\varepsilon}}, & \forall p \in H^{\frac{1}{2}-\varepsilon}(C), \\
\inf _{\mu_{H} \in \mathcal{G}_{H}}\left\|\lambda-\mu_{H}\right\|_{\mathcal{G}} \leq C H^{1 / 2-\varepsilon}\|\lambda\|_{H^{1-\varepsilon}(\Gamma)}, & \forall \lambda \in H^{1-\varepsilon}(\Gamma),
\end{aligned}\right.
$$

and this finally implies the following error estimates with respect to $h$.

Corollary 4.2. Assume that

$$
(\mathbf{v}, p, \lambda) \in \mathcal{C}^{k}\left([0, T] ; H^{\frac{1}{2}-\varepsilon}(\operatorname{div}, C) \times H^{\frac{1}{2}-\varepsilon}(C) \times H^{1-\varepsilon}(\Gamma)\right), \quad \varepsilon>0
$$

and that $H \geq \alpha$ where $\alpha$ is the constant given in Theorem 4.1. Then we have the estimates

$$
\begin{aligned}
\left\|\partial_{t}^{k}\left(\widehat{\mathbf{v}_{h}}-\mathbf{v}\right)\right\|_{\underline{X}}+\left\|\partial_{t}^{k}\left(\widehat{\lambda}_{H}-\lambda\right)\right\|_{\mathcal{G}} \leq & \mathcal{C}\left(h^{\frac{1}{2}-\varepsilon}\left\|\partial_{t}^{k} \mathbf{v}\right\|_{H_{\mathrm{div}}^{\frac{1}{2}-\varepsilon}}+H^{\frac{1}{2}-\varepsilon}\left\|\partial_{t}^{k} \lambda\right\|_{H^{1-\varepsilon}(\Gamma)}\right) \\
\left\|P_{M_{h}^{0}}\left(\partial_{t}^{k} \widehat{p}_{h}\right)-\partial_{t}^{k} p\right\|_{M}+\left\|P_{M_{h}^{r}}\left(\partial_{t}^{k} \widehat{p}_{h}\right)\right\|_{M} \leq & \mathcal{C}\left(h^{\frac{1}{2}-\varepsilon}\left(\left\|\partial_{t}^{k} \mathbf{v}\right\|_{H_{\mathrm{div}}^{\frac{1}{2}-\varepsilon}(C)}+\left\|\partial_{t}^{k} p\right\|_{H^{\frac{1}{2}-\varepsilon}(C)}\right)\right. \\
& \left.+H^{\frac{1}{2}-\varepsilon}\left\|\partial_{t}^{k} \lambda\right\|_{H^{1-\varepsilon}(\Gamma)}\right) .
\end{aligned}
$$

\subsection{Error estimates}

The error estimates for the evolution problem are then quite standard. They follow from energy estimates. We define the discrete energy of the error as

$$
\mathcal{E}_{h}=\left\|\widehat{p}_{h}-p_{h}\right\|_{\rho}^{2}+a\left(\widehat{\mathbf{v}}_{h}-\mathbf{v}_{h}, \widehat{\mathbf{v}}_{h}-\mathbf{v}_{h}\right) .
$$


We first prove the energy identity:

Theorem 4.4. The discrete energy of the error satisfies the identity,

$$
\frac{\mathrm{d} \mathcal{E}_{h}}{\mathrm{~d} t}+\int_{C} \beta\left|\widehat{p}_{h}^{r}-p_{h}^{r}\right|^{2}=F
$$

where

$$
\begin{aligned}
F= & \frac{\mathrm{d}}{\mathrm{d} t}\left(\left(\widehat{p}_{h}-p, \widehat{p}_{h}-p_{h}\right)_{\rho}+a\left(\widehat{\mathbf{v}}_{h}-\mathbf{v}, \widehat{\mathbf{v}}_{h}-\mathbf{v}_{h}\right)\right) \\
& -\left(\widehat{p}_{h}-p, \widehat{p}_{h}-p_{h}\right)_{\rho}-a\left(\widehat{\mathbf{v}}_{h}-\mathbf{v}, \widehat{\mathbf{v}}_{h}-\mathbf{v}_{h}\right)+\int_{C} \beta \widehat{p}_{h}^{r}\left(\widehat{p}_{h}^{r}-p_{h}^{r}\right) .
\end{aligned}
$$

Proof. The difference between the continuous problem (1.7) and the discrete one (3.5) gives a problem satisfied by the error

$$
\left(\mathbf{v}_{h}-\mathbf{v}, p_{h}-p, \lambda_{H}-\lambda\right)=\left(\widehat{\mathbf{v}}_{h}-\mathbf{v}, \widehat{p}_{h}-p, \widehat{\lambda}_{H}-\lambda\right)+\left(\mathbf{v}_{h}-\widehat{\mathbf{v}}_{h}, p_{h}-\widehat{p}_{h}, \lambda_{H}-\widehat{\lambda}_{H}\right) .
$$

Using the definition of the elliptic projection operator, all the terms which would give rise to difficulties in obtaining the energy estimate (essentially those that are not equivalent to $L^{2}$ norms) disappear. It remains; $\forall\left(q_{h}, \mathbf{w}_{h}, \mu_{H}\right) \in M_{h} \times \underline{X_{h}} \times \mathcal{G}_{H}:$

$$
\left\{\begin{array}{l}
\frac{\mathrm{d}}{\mathrm{d} t}\left(\widehat{p}_{h}-p_{h}, q_{h}\right)_{\rho}-\left(\beta p_{h}^{r}, q_{h}\right)-b\left(\left(\widehat{\mathbf{v}}_{h}-\mathbf{v}_{h}\right), q_{h}\right)=\frac{\mathrm{d}}{\mathrm{d} t}\left(\widehat{p}_{h}-p, q_{h}\right)_{\rho}-\left(\widehat{p}_{h}-p, q_{h}\right)_{\rho}, \\
\frac{\mathrm{d}}{\mathrm{d} t} a\left(\widehat{\mathbf{v}}_{h}-\mathbf{v}_{h}, \mathbf{w}_{h}\right)+b\left(\mathbf{w}_{h}, \widehat{p}_{h}-p_{h}\right)-\left\langle\widehat{\mathbf{v}}_{h} \cdot \mathbf{n}, \widehat{\lambda}_{H}-\lambda_{H}\right\rangle_{\Gamma}=\frac{\mathrm{d}}{\mathrm{d} t} a\left(\widehat{\mathbf{v}}_{h}-\mathbf{v}, \mathbf{w}_{h}\right)-a\left(\widehat{\mathbf{v}}_{h}-\mathbf{v}, \mathbf{w}_{h}\right), \\
\left\langle\left(\widehat{\mathbf{v}}_{h}-\mathbf{v}_{h}\right) \cdot \mathbf{n}, \mu_{H}\right\rangle_{\Gamma}=0 .
\end{array}\right.
$$

For $q_{h}=\widehat{p}_{h}-p_{h}$ and $\mathbf{w}_{h}=\widehat{\mathbf{v}}_{h}-\mathbf{v}_{h}$ we obtain

$$
\left\{\begin{array}{l}
\frac{\mathrm{d}}{\mathrm{d} t}\left\|\widehat{p}_{h}-p_{h}\right\|_{\rho}^{2}-\left(p_{h}^{r}, \widehat{p}_{h}-p_{h}\right)_{\beta}-b\left(\left(\widehat{\mathbf{v}}_{h}-\mathbf{v}_{h}\right), \widehat{p}_{h}-p_{h}\right)=\frac{\mathrm{d}}{\mathrm{d} t}\left(\widehat{p}_{h}-p, \widehat{p}_{h}-p_{h}\right)_{\rho}-\left(\widehat{p}_{h}-p, \widehat{p}_{h}-p_{h}\right)_{\rho}, \\
\frac{\mathrm{d}}{\mathrm{d} t} a\left(\widehat{\mathbf{v}}_{h}-\mathbf{v}_{h}, \widehat{\mathbf{v}}_{h}-\mathbf{v}_{h}\right)+b\left(\left(\widehat{\mathbf{v}}_{h}-\mathbf{v}_{h}\right), \widehat{p}_{h}-p_{h}\right)-\left\langle\left(\widehat{\mathbf{v}}_{h}-\mathbf{v}_{h}\right) \cdot \mathbf{n}, \widehat{\lambda}_{H}-\lambda_{H}\right\rangle_{\Gamma}= \\
\frac{\mathrm{d}}{\mathrm{d} t} a\left(\widehat{\mathbf{v}}_{h}-\mathbf{v}, \widehat{\mathbf{v}}_{h}-\mathbf{v}_{h}\right)-a\left(\widehat{\mathbf{v}}_{h}-\mathbf{v}, \widehat{\mathbf{v}}_{h}-\mathbf{v}_{h}\right), \\
\left\langle\left(\widehat{\mathbf{v}}_{h}-\mathbf{v}_{h}\right) \cdot \mathbf{n}, \mu_{H}\right\rangle_{\Gamma}=0, \quad \forall \mu_{H} \in \mathcal{G}_{H} .
\end{array}\right.
$$

Adding the first two equations and using the third one gives (4.15).

The following proposition gives a bound of the discrete energy of the error in terms of the elliptic projection error.

Proposition 4.1. The discrete energy of the error satisfies the following estimate:

$$
\begin{aligned}
\sup _{t^{\prime} \leq T} \mathcal{E}_{h}^{\frac{1}{2}}\left(t^{\prime}\right) \leq & \mathcal{C} \mathcal{E}_{h}^{\frac{1}{2}}(0)+\mathcal{C} \int_{0}^{T}\left(\left\|\partial_{t}\left(\widehat{p}_{h}-p\right)\right\|_{M}+\left\|\partial_{t}\left(\widehat{\mathbf{v}}_{h}-\mathbf{v}\right)\right\|_{L^{2}}(C)\right. \\
& \left.+\left\|\widehat{p}_{h}-p\right\|_{M}+\left\|\widehat{\mathbf{v}}_{h}-\mathbf{v}\right\|_{\underline{L^{2}}(C)}\right) \mathrm{d} s+\mathcal{C}\left[\int_{0}^{T}\left\|\sqrt{\beta} P_{M_{h}^{r}}\left(\widehat{p}_{h}\right)\right\|_{M}^{2} \mathrm{~d} s\right]^{\frac{1}{2}},
\end{aligned}
$$

where $\mathcal{C}$ is a constant independent of $h$ and $\beta$. 
Proof. The proof is based on equality (4.15). Due to Young's inequality, the last term in (4.16) can be bounded by:

$$
\int_{C}\left|\beta P_{M_{h}^{r}}\left(\widehat{p}_{h}\right) P_{M_{h}^{r}}\left(\widehat{p}_{h}-p_{h}\right)\right| \mathrm{d} x \leq \int_{C} \beta\left|P_{M_{h}^{r}}\left(\widehat{p}_{h}-p_{h}\right)\right|^{2} \mathrm{~d} x+\int_{C} \frac{\beta}{4}\left|P_{M_{h}^{r}}\left(\widehat{p}_{h}\right)\right|^{2} \mathrm{~d} x .
$$

Simple computations then give

$$
\begin{aligned}
\frac{\mathrm{d}}{\mathrm{d} t} \mathcal{E}_{h}(t) \leq & \mathcal{C}\left\|\widehat{p}_{h}-p_{h}\right\|_{M}\left(\left\|\partial_{t}\left(\widehat{p}_{h}-p\right)\right\|_{M}+\left\|\widehat{p}_{h}-p\right\|_{M}\right) \\
& +\mathcal{C}\left\|\widehat{\mathbf{v}}_{h}-\mathbf{v}_{h}\right\|_{\underline{L^{2}}(C)}\left(\left\|\partial_{t}\left(\widehat{\mathbf{v}}_{h}-\mathbf{v}\right)\right\|_{\underline{L}^{2}(C)}+\left\|\widehat{\mathbf{v}}_{h}-\mathbf{v}\right\|_{\underline{L^{2}(C)}}\right)+\frac{1}{4} \| \sqrt{\beta} P_{M_{h}^{r}\left(\widehat{p}_{h}\right) \|_{M}^{2}} \\
\leq & \mathcal{C} \mathcal{E}_{h}^{\frac{1}{2}}(t)\left(\left\|\partial_{t}\left(\widehat{p}_{h}-p\right)\right\|_{M}+\left\|\widehat{p}_{h}-p\right\|_{M}+\left\|\partial_{t}\left(\widehat{\mathbf{v}}_{h}-\mathbf{v}\right)\right\|_{L^{2}(C)}+\left\|\widehat{\mathbf{v}}_{h}-\mathbf{v}\right\|_{L^{2}(C)}\right) \\
& +\frac{1}{4}\left\|\sqrt{\beta} P_{M_{h}^{r}}\left(\widehat{p}_{h}\right)\right\|_{M}^{2} .
\end{aligned}
$$

Integrating in time, we obtain $(\forall t \leq T)$

$$
\begin{aligned}
\mathcal{E}_{h}(t) \leq \mathcal{E}_{h}(0)+\mathcal{C} \sup _{t^{\prime} \leq T} & \mathcal{E}_{h}^{\frac{1}{2}}\left(t^{\prime}\right) \int_{0}^{T}\left(\left\|\partial_{t}\left(\widehat{p}_{h}-p\right)\right\|_{M}+\left\|\partial_{t}\left(\widehat{\mathbf{v}}_{h}-\mathbf{v}\right)\right\|_{\underline{L^{2}}(C)}\right. \\
& \left.+\left\|\widehat{p}_{h}-p\right\|_{M}+\left\|\widehat{\mathbf{v}}_{h}-\mathbf{v}\right\|_{\underline{L^{2}}(C)}\right) \mathrm{d} s+\frac{1}{4} \int_{0}^{T}\left\|\sqrt{\beta} P_{M_{h}^{r}}\left(\widehat{p}_{h}\right)\right\|_{M}^{2} \mathrm{~d} s .
\end{aligned}
$$

We then take the maximum on $t \leq T$ and apply Young's inequality to the first integral term:

$$
\begin{aligned}
\sup _{t^{\prime} \leq T} \mathcal{E}_{h}\left(t^{\prime}\right) \leq \mathcal{C} & \mathcal{E}_{h}(0)+\mathcal{C}\left[\int _ { 0 } ^ { T } \left(\left\|\partial_{t}\left(\widehat{p}_{h}-p\right)\right\|_{M}+\left\|\partial_{t}\left(\widehat{\mathbf{v}}_{h}-\mathbf{v}\right)\right\|_{L^{2}(C)}\right.\right. \\
& \left.\left.+\left\|\widehat{p}_{h}-p\right\|_{M}+\left\|\widehat{\mathbf{v}}_{h}-\mathbf{v}\right\|_{\underline{L^{2}}(C)}\right) \mathrm{d} s\right]^{2}+\mathcal{C} \int_{0}^{T}\left\|\sqrt{\beta} P_{M_{h}^{r}}\left(\widehat{p}_{h}\right)\right\|_{M}^{2} \mathrm{~d} s
\end{aligned}
$$

which easily implies (4.17).

We can now give error estimates:

Theorem 4.5. Let $f \in C^{0}([0, T], M), \mathbf{v}_{0} \in \underline{X}, p_{0} \in M$ satisfy (1.3) and let $(\mathbf{v}, p, \lambda)$ be the solution of problem (1.7) defined in Theorem 1.2. Let $\left(\mathbf{v}_{h}, p_{h}, \lambda_{H}\right)$ be the solution of (3.5) with initial data $\left(\mathbf{v}_{h, 0}, p_{h, 0}\right)$, the two first components of $\Pi_{h}\left(\mathbf{v}_{0}, p_{0}, 0\right)$. Then, we have the error estimates

$$
\begin{aligned}
& \left\|\mathbf{v}_{h}-\mathbf{v}\right\|_{C^{0}\left([0, T] ; \underline{L^{2}}(C)\right)}+\left\|p_{h}-p\right\|_{C^{0}([0, T] ; M)} \leq \\
& \mathcal{C}(1+T)\left(\left\|\widehat{\mathbf{v}}_{h}-\mathbf{v}\right\|_{C^{1}\left([0, T] ; \underline{\left.L^{2}(C)\right)}\right.}+\left\|\widehat{p}_{h}-p\right\|_{C^{1}([0, T] ; M)}\right) \\
& +\mathcal{C} \sqrt{T}\|\sqrt{\beta}\|_{L^{\infty}(C)}\left\|P_{M_{h}^{r}}\left(\widehat{p}_{h}\right)\right\|_{C^{0}([0, T] ; M)} .
\end{aligned}
$$


Furthermore, if $(\mathbf{v}, p) \in C^{2}\left([0, T] ; \underline{L^{2}}(C) \times M\right)$ then

$$
\begin{aligned}
\left\|\mathbf{v}_{h}-\mathbf{v}\right\|_{C^{0}([0, T] ; \underline{X})} \leq \mathcal{C} & \left\|\widehat{\mathbf{v}}_{h}-\mathbf{v}\right\|_{C^{0}([0, T] ; \underline{X})} \\
& +\mathcal{C}(1+T)\left(1+\|\beta\|_{L^{\infty}(C)}\right)\left(\left\|\widehat{\mathbf{v}}_{h}-\mathbf{v}\right\|_{C^{2}\left([0, T] ; L^{2}(C)\right)}+\left\|\widehat{p}_{h}-p\right\|_{C^{2}([0, T] ; M)}\right) \\
& +\|\beta\|_{L^{\infty}(C)}\left(1+\sqrt{T}\|\sqrt{\beta}\|_{L^{\infty}}\right)\left\|P_{M_{h}^{r}}\left(\widehat{p}_{h}\right)\right\|_{C^{1}([0, T] ; M)},
\end{aligned}
$$

$$
\begin{aligned}
\left\|\lambda_{H}-\lambda\right\|_{C^{0}([0, T] ; \mathcal{G}) \leq \mathcal{C}\left\|\widehat{\lambda_{H}}-\lambda\right\|_{C^{0}([0, T] ; \mathcal{G})}} \\
+\mathcal{C}(1+T)\left(\left\|\widehat{\mathbf{v}}_{h}-\mathbf{v}\right\|_{C^{2}\left([0, T] ; \underline{L^{2}}(C)\right)}+\left\|\widehat{p}_{h}-p\right\|_{C^{2}([0, T] ; M)}\right) \\
+\mathcal{C} \sqrt{T}\|\sqrt{\beta}\|_{L^{\infty}}\left\|P_{M_{h}^{r}}\left(\widehat{p}_{h}\right)\right\|_{C^{1}([0, T] ; M)} .
\end{aligned}
$$

Proof. First, notice that the choice of the approximate initial data implies $\mathcal{E}_{h}(0)=0$. Then the inequality (4.17) easily implies (4.18). This gives an error estimate for $\mathbf{v}$ in the $L^{2}$ norm. In order to obtain an estimate in the $\underline{X}$ norm, we first state a similar result for the time derivative of the solution. Assuming that the solution is one order more regular, then

$$
\begin{aligned}
\left\|\partial_{t}\left(\mathbf{v}_{h}-\mathbf{v}\right)\right\|_{C^{0}\left([0, T] ; L^{2}(C)\right)}+\left\|\partial_{t}\left(p_{h}-p\right)\right\|_{C^{0}([0, T] ; M)} \leq & \\
\mathcal{C}(1+T)\left(\left\|\partial_{t}\left(\widehat{\mathbf{v}}_{h}-\mathbf{v}\right)\right\|_{C^{1}\left([0, T] ; \underline{L^{2}}(C)\right)}\right. & \left.+\left\|\partial_{t}\left(\widehat{p}_{h}-p\right)\right\|_{C^{1}([0, T] ; M)}\right) \\
& +\mathcal{C} \sqrt{T}\|\sqrt{\beta}\|_{L^{\infty}(C)}\left\|P_{M_{h}^{r}}\left(\partial_{t} \widehat{p}_{h}\right)\right\|_{C^{0}([0, T] ; M)} .
\end{aligned}
$$

Subtracting from the second equation of (3.5) the second equation of (1.7) we obtain

$$
\left(\operatorname{div}\left(\mathbf{v}_{h}-\mathbf{v}\right), q_{h}\right)=\left(\partial_{t}\left(p_{h}-p\right), q_{h}\right)_{\rho}+\left(\beta P_{M_{h}^{r}}\left(p_{h}\right), q_{h}\right), \quad \forall q_{h} \in M_{h} .
$$

This implies $\left(\operatorname{div}\left(\mathbf{v}_{h}-\widehat{\mathbf{v}}_{h}\right) \in M_{h}\right)$

$$
\left\|\operatorname{div}\left(\mathbf{v}_{h}-\widehat{\mathbf{v}}_{h}\right)\right\|_{L^{2}(C)} \leq \mathcal{C}\left(\left\|\partial_{t}\left(p_{h}-p\right)\right\|_{M}+\left\|\operatorname{div}\left(\widehat{\mathbf{v}}_{h}-\mathbf{v}\right)\right\|_{L^{2}(C)}+\left\|\beta P_{M_{h}^{r}}\left(p_{h}\right)\right\|_{M}\right),
$$

and therefore

$$
\begin{aligned}
\left\|\operatorname{div}\left(\mathbf{v}_{h}-\mathbf{v}\right)\right\|_{L^{2}(C)} \leq \mathcal{C}\left(\left\|\partial_{t}\left(p_{h}-p\right)\right\|_{M}+\left\|\widehat{\mathbf{v}}_{h}-\mathbf{v}\right\|_{\underline{X}}\right) & \\
& +\mathcal{C}\|\beta\|_{L^{\infty}(C)}\left(\left\|\widehat{p}_{h}-p\right\|_{M}+\left\|p_{h}-p\right\|_{M}+\left\|P_{M_{h}^{r}}\left(\widehat{p}_{h}\right)\right\|_{M}\right) .
\end{aligned}
$$

Then using (4.18), (4.21) and this last equation we obtain (4.19). It remains to obtain the estimate for the Lagrange multiplier. Due to the uniform discrete inf-sup condition (4.4) applied to $\lambda_{H}-\widehat{\lambda_{H}}$, there exists $\mathbf{w}_{h} \in \underline{X}_{h}$ such that

$$
\begin{aligned}
\mathcal{C}\left\|\lambda_{H}-\widehat{\lambda_{H}}\right\|_{\mathcal{G}}\left\|\mathbf{w}_{h}\right\|_{\underline{X}} & \leq\left\langle\mathbf{w}_{h} \cdot \mathbf{n}, \lambda_{H}-\widehat{\lambda_{H}}\right\rangle_{\Gamma} \\
& =\left\langle\mathbf{w}_{h} \cdot \mathbf{n}, \lambda_{H}-\lambda\right\rangle_{\Gamma}+\left\langle\mathbf{w}_{h} \cdot \mathbf{n}, \lambda-\widehat{\lambda_{H}}\right\rangle_{\Gamma} \\
& =a\left(\partial_{t}\left(\mathbf{v}_{h}-\mathbf{v}\right), \mathbf{w}_{h}\right)+b\left(\mathbf{w}_{h}, p_{h}-p\right)+\left\langle\mathbf{w}_{h} \cdot \mathbf{n}, \lambda-\widehat{\lambda_{H}}\right\rangle_{\Gamma} .
\end{aligned}
$$

This implies that

$$
\left\|\lambda_{H}-\lambda\right\|_{\mathcal{G}} \leq \mathcal{C}\left(\left\|\lambda-\widehat{\lambda_{H}}\right\|_{\mathcal{G}(\Gamma)}+\left\|\partial_{t}\left(\mathbf{v}_{h}-\mathbf{v}\right)\right\|_{L^{2}(C)}+\left\|p_{h}-p\right\|_{M}\right),
$$

and therefore, using (4.18) and (4.21) we show estimate (4.20). 

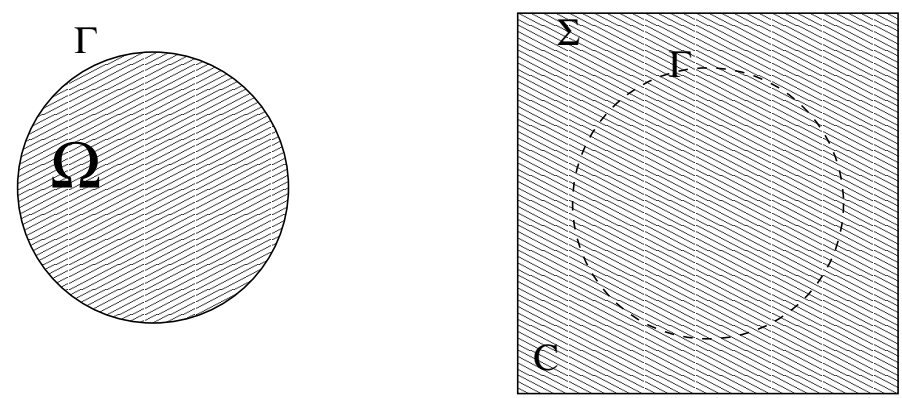

FIgURE 6 . The geometry of the problem. On the left the initial domain of propagation $\Omega$ and on the right the extended domain, $C$, introduced by the fictitious domain formulation of the problem.

Finally, the following theorem gives the order of convergence of the method.

Theorem 4.6. We make the same assumptions as in Theorem 4.5. Then we have

- If $(\mathbf{v}, p, \lambda) \in C^{1}\left([0, T] ; H^{\frac{1}{2}-\varepsilon}(\operatorname{div}, C) \times H^{\frac{1}{2}-\varepsilon}(C) \times H^{1-\varepsilon}(\Gamma)\right)$ then

$$
\begin{gathered}
\left\|\mathbf{v}_{h}-\mathbf{v}\right\|_{C^{0}\left([0, T] ; \underline{L^{2}}(C)\right)}+\left\|p_{h}-p\right\|_{C^{0}([0, T] ; M)} \leq \mathcal{C}\left(h^{\frac{1}{2}-\varepsilon}+H^{\frac{1}{2}-\varepsilon}\right) \\
{\left[(1+T)\left(\|\mathbf{v}\|_{C^{1}\left([0, T] ; H_{\text {div }}^{\frac{1}{2}-\varepsilon}(C)\right)}+\|p\|_{C^{1}\left([0, T] ; H^{\frac{1}{2}-\varepsilon}(C)\right)}+\|\lambda\|_{\left.C^{1}\left([0, T] ; H^{1-\varepsilon}(\Gamma)\right)\right)}\right)\right.} \\
\left.+\sqrt{T}\|\sqrt{\beta}\|_{L^{\infty}(C)}\left(\|\mathbf{v}\|_{C^{0}\left([0, T] ; H_{\text {div }}^{\frac{1}{2}-\varepsilon}(C)\right)}+\|p\|_{C^{0}\left([0, T] ; H^{\frac{1}{2}-\varepsilon}(C)\right)}+\|\lambda\|_{\left.C^{0}\left([0, T] ; H^{1-\varepsilon}(\Gamma)\right)\right)}\right)\right] .
\end{gathered}
$$

- If $(\mathbf{v}, p, \lambda) \in C^{2}\left([0, T] ; H^{\frac{1}{2}-\varepsilon}(\operatorname{div}, C) \times H^{\frac{1}{2}-\varepsilon}(C) \times H^{1-\varepsilon}(\Gamma)\right)$ then

$$
\begin{aligned}
\left\|\mathbf{v}_{h}-\mathbf{v}\right\|_{C^{0}([0, T] ; \underline{X})} \leq \mathcal{C}\left(h^{\frac{1}{2}-\varepsilon}+H^{\frac{1}{2}-\varepsilon}\right)(1+T)\left(1+\left\|\beta^{\frac{3}{2}}\right\|_{L^{\infty}(C)}\right) \\
\times\left(\|\mathbf{v}\|_{C^{2}\left([0, T] ; H_{\left.\operatorname{div}^{\frac{1}{2}-\varepsilon}(C)\right)}\right.}+\|p\|_{C^{2}\left([0, T] ; H^{\frac{1}{2}-\varepsilon}(C)\right)}+\|\lambda\|_{C^{2}\left([0, T] ; H^{1-\varepsilon}(\Gamma)\right)}\right),
\end{aligned}
$$

$\left\|\lambda_{H}-\lambda\right\|_{C^{0}([0, T] ; \mathcal{G})} \leq \mathcal{C}\left(h^{\frac{1}{2}-\varepsilon}+H^{\frac{1}{2}-\varepsilon}\right)$

$$
\begin{gathered}
\times\left[(1+T)\left(\|\mathbf{v}\|_{C^{2}\left([0, T] ; H_{\text {div }}^{\frac{1}{2}-\varepsilon}(C)\right)}+\|p\|_{C^{2}\left([0, T] ; H^{\frac{1}{2}-\varepsilon}(C)\right)}+\|\lambda\|_{C^{2}\left([0, T] ; H^{1-\varepsilon}(\Gamma)\right)}\right)\right. \\
\left.+\sqrt{T}\|\sqrt{\beta}\|_{L^{\infty}(C)}\left(\|\mathbf{v}\|_{C^{1}\left([0, T] ; H_{\operatorname{div}}^{\frac{1}{2}-\varepsilon}(C)\right)}+\|p\|_{C^{1}\left([0, T] ; H^{\frac{1}{2}-\varepsilon}(C)\right)}+\|\lambda\|_{C^{1}\left([0, T] ; H^{1-\varepsilon}(\Gamma)\right)}\right)\right] .
\end{gathered}
$$

Proof. This is a consequence of estimates on the evolution problem obtained in Theorem 4.5 combined with the estimates obtained on the elliptic projection error in Corollary 4.2.

\section{Numerical ERror estimates}

In this section we are interested in confirming numerically the order of convergence of the method. To do so, we consider solving the wave equation on a disk $\Omega \subset \mathbb{R}^{2}$ with homogeneous Neumann boundary conditions on its boundary $\Gamma=\partial \Omega$. The geometry of the problem is presented in Figure 6 .

To compute the solution we extend the unknowns to the domain of simple geometry $C$ (see Fig. 6 ) and use the fictitious domain formulation (1.7) with a zero force term $f=0$ and the initial conditions given in Section 2.2. The center of the initial condition, $\left(x_{c}, z_{c}\right)=(5,5) \mathrm{mm}$, coincides with the center of the disk $\Omega$ whose radius 
is $R=4 \mathrm{~mm}$. The physical properties of the material and size of the computational domain are the same as in Section 2.2. In practice to truncate the extended domain $C$, we surround the computational domain by a perfectly matched absorbing layer model (PML $[9,11])$.

We remark that the solution of this problem is rotationally invariant because of the symmetry in the geometry and the initial conditions. We use this symmetry in order to compute a reference solution by solving a one dimensional problem. More precisely, when expressed in cylindrical coordinates, it is easy to see that the solution of the two dimensional problem $\left(\Omega\right.$ being $[0, R] \times[0,2 \pi]$, and where $\varrho=1000 \mathrm{Kg} \cdot \mathrm{m}^{-3}$ and $a=10^{9} \mathrm{~Pa}$ )

$$
\begin{cases}a \frac{\partial v_{r}}{\partial t}-\frac{\partial p}{\partial r}=0, & \text { in }[0, R] \times[0,2 \pi], \\ a \frac{\partial v_{\theta}}{\partial t}-\frac{\partial p}{\partial \theta}=0, & \text { in }[0, R] \times[0,2 \pi], \\ \varrho \frac{\partial p}{\partial t}-\frac{\partial v_{r}}{\partial r}-\frac{1}{r} v_{r}=0, & \text { in }[0, R] \times[0,2 \pi], \\ v_{r}=0, & \text { on }[r=R] \times[0,2 \pi],\end{cases}
$$

with initial conditions,

$$
p_{0}(r, \theta)=0.1 F\left(r / r_{0}\right), v_{r}=v_{\theta}=0
$$

depends only on $r$, i.e., $v_{r}(r, \theta)=v_{r}(r), v_{\theta}=0, p(r, \theta)=p(r)$. Thus, it can be deduced by solving the following one-dimensional problem,

with initial conditions,

$$
\begin{cases}a \frac{\partial v_{r}}{\partial t}-\frac{\partial p}{\partial r}=0, & \text { in }[0, R], \\ \varrho \frac{\partial p}{\partial t}-\frac{\partial v_{r}}{\partial r}-\frac{1}{r} v_{r}=0, & \text { in }[0, R], \\ v_{r}=0, & \text { for } r=0 \text { and } r=R,\end{cases}
$$

To solve numerically the one-dimensional problem (5.1), we use piecewise constant functions for the discretization of $p_{r}$ and continuous piecewise linear functions for $v_{r}$. For the time discretization a second order leap frog scheme is employed.

In Figure 7 we display the results of the numerical convergence analysis. The reference solution in $1 \mathrm{D}$, is obtained on a fine grid with a spatial discretization step $h_{1 d}=1 / 160 \mathrm{~mm}$. The two dimensional problem is solved with four different discretizations using $h_{x}=h_{z}=h=1 / 10,1 / 20,1 / 40$ and $1 / 80 \mathrm{~mm}$ and $H=1.2 h$. For each discretization we compute the difference between the obtained solution and the reference one. In Figure 7 we display the logarithm of the error as a function of the logarithm of the discretization step. The rate of convergence is deduced from the slope $s$ of the line. We can remark that the results obtained numerically are slightly better than our theoretical predictions. Note however, that the estimate obtained on the $L^{\infty}([0, T], H(\operatorname{div}))$ norm of $\mathbf{v}$ is $h^{0.48}$ which indicates that the theoretical estimations are optimal.

In Figure 8 we display the same results but with the norm of the error now computed in $\widetilde{C}=C / B_{b}(\Gamma)$, i.e., the domain $C$ restricted away from $B_{b}(\Gamma)$, which is defined by

$$
B_{b}(\Gamma)=\left\{\mathbf{x} \in C \text { s.t. } \min _{\mathbf{y} \in \Gamma}|\mathbf{x}-\mathbf{y}| \leq b\right\} .
$$

In this case, we observe that the convergence rate of the method is higher. Furthermore, we noticed numerically that $b=h$ is the critical value, i.e., the convergence rate does not change for bigger values of $b$ and it decreases for $b<h$. This agrees with our intuition in the sense that the elements that we need to remove are the ones in which the solution has less regularity (see Rem. 1.2), i.e., the elements that have non-zero intersection with the boundary $\Gamma$. 


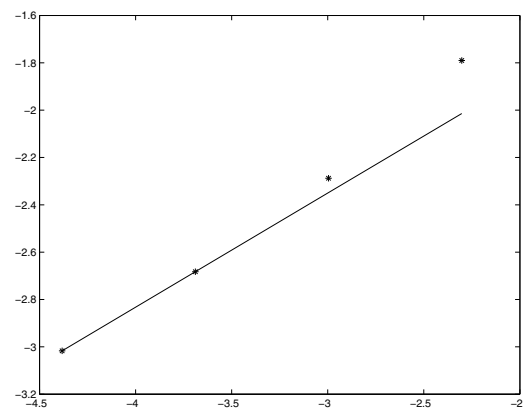

(a) $\sup _{t \leq T}\left\|\mathbf{v}-\mathbf{v}_{h}\right\|_{\underline{X}}, s=0.48$

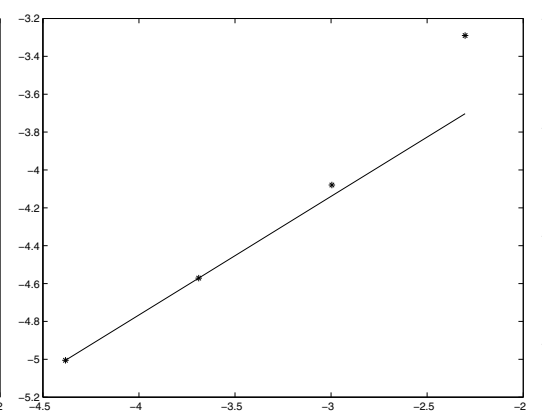

(b) $\sup _{t \leq T}\left\|p-p_{h}\right\|_{M}, s=0.63$.

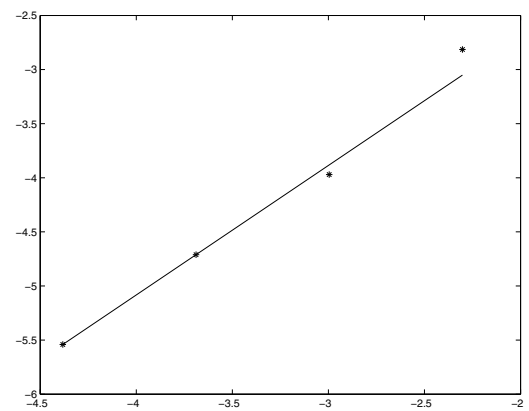

(c) $\sup _{t \leq T}\left\|\lambda-\lambda_{h}\right\|_{L^{2}}, s=1.2$.

FiguRE 7. Numerical error on $\mathbf{v}, p$ and $\lambda$ versus the discretization step.

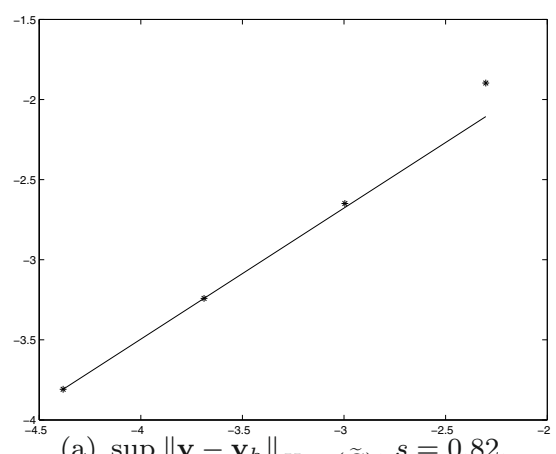

(a) $\sup _{t \leq T}^{-4}\left\|\mathbf{v}-\mathbf{v}_{h}\right\|_{\underline{H_{\text {div }}}}{ }^{-3}(\widetilde{C}), s \stackrel{-2.5}{=} 0.82$.

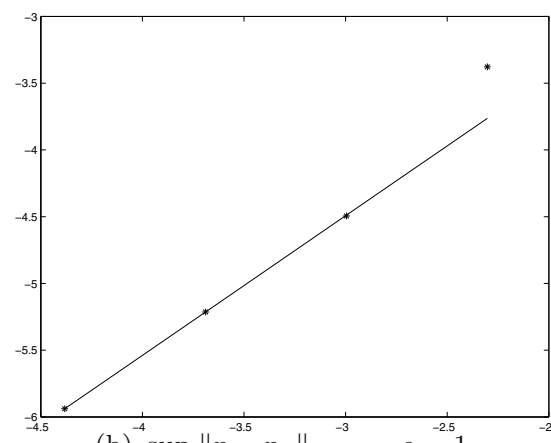

(b) $\sup _{t \leq T}^{-4}\left\|p^{-3.5}-p_{h}\right\|_{L^{2}(\widetilde{C})}^{-3}, s \stackrel{-2.5}{=} 1$.

Figure 8. Numerical error on $\mathbf{v}, p$ and $\lambda$ versus the discretization step. Here we compute the norm of the error in the domain $\widetilde{C}$ which is $C$ restricted from $B_{b}(\Gamma)$, i.e., $\Gamma$ and its vicinity $(5.2)$.

Finally, notice that the convergence rate on $\lambda$ (approximately 1 ) is computed in the $L^{2}(\Gamma)$ norm and therefore we recover the expected convergence rate $(1 / 2)$ in $H^{1 / 2}(\Gamma)$.

\section{CONCLUSiOn}

We considered in this paper the application of the fictitious domain method while taking into account the Neumann boundary condition on the surface of an object in the context of acoustic wave propagation. We first demonstrated with numerical examples that the method introduced in [4] does not converge for all crack geometries. We proposed instead the use of a modified version of the mixed finite elements introduced in [3]. Those elements were obtained by enriching the approximation space for the pressure field while keeping the same space for the velocity. Due to this enrichment spurious propagating modes (non-physical waves) are introduced in the discrete solution. To damp these spurious modes we proposed a discrete method that introduces artificial absorption of the non-physical waves only. We carried out the theoretical convergence analysis of the method and obtained error estimates. For a particular object geometry we also performed a numerical study of the convergence showing that our theoretical error estimates are optimal. 


\section{REFERENCES}

[1] I. Babuška, The finite element method with lagrangian multipliers. Numer. Math. 20 (1973) 179-192.

[2] E. Bécache, P. Joly and C. Tsogka, Éléments finis mixtes et condensation de masse en élastodynamique linéaire, (i) Construction. C. R. Acad. Sci. Paris Sér. I 325 (1997) 545-550.

[3] E. Bécache, P. Joly and C. Tsogka, An analysis of new mixed finite elements for the approximation of wave propagation problems. SINUM 37 (2000) 1053-1084.

[4] E. Bécache, P. Joly and C. Tsogka, Fictitious domains, mixed finite elements and perfectly matched layers for $2 \mathrm{~d}$ elastic wave propagation. J. Comp. Acoust. 9 (2001) 1175-1203.

[5] E. Bécache, P. Joly and C. Tsogka, A new family of mixed finite elements for the linear elastodynamic problem. SINUM 39 (2002) 2109-2132.

[6] E. Bécache, A. Chaigne, G. Derveaux and P. Joly, Time-domain simulation of a guitar: Model and method. J. Acoust. Soc. Am. 6 (2003) 3368-3383.

[7] E. Bécache, J. Rodríguez and C. Tsogka, On the convergence of the fictitious domain method for wave equation problems. Technical Report RR-5802, INRIA (2006).

[8] E. Bécache, J. Rodríguez and C. Tsogka, A fictitious domain method with mixed finite elements for elastodynamics. SIAM J. Sci. Comput. 29 (2007) 1244-1267.

[9] J.P. Bérenger, A perfectly matched layer for the absorption of electromagnetic waves. J. Comp. Phys. 114 (1994) 185-200.

[10] F. Brezzi and M. Fortin, Mixed and Hybrid Finite Element Methods. Springer-Verlag, New York (1991).

[11] F. Collino and C. Tsogka, Application of the PML absorbing layer model to the linear elastodynamic problem in anisotropic heteregeneous media. Geophysics 66 (2001) 294-307.

[12] F. Collino, P. Joly and F. Millot, Fictitious domain method for unsteady problems: Application to electromagnetic scattering. J. Comput. Phys. 138 (1997) 907-938.

[13] S. Garcès, Application des méthodes de domaines fictifs à la modélisation des structures rayonnantes tridimensionnelles. Ph.D. Thesis, SupAero, France (1998).

[14] V. Girault and R. Glowinski, Error analysis of a fictitious domain method applied to a Dirichlet problem. Japan J. Indust. Appl. Math. 12 (1995) 487-514.

[15] V. Girault and P.-A. Raviart, Finite element methods for Navier-Stokes equations - Theory and algorithms, Springer Series in Computational Mathematics 5. Springer-Verlag, Berlin (1986).

[16] R. Glowinski, Numerical methods for fluids, Part 3, Chapter 8, in Handbook of Numerical Analysis IX, P.G. Ciarlet and J.L. Lions Eds., North-Holland, Amsterdam (2003) x+1176.

[17] R. Glowinski and Y. Kuznetsov, On the solution of the Dirichlet problem for linear elliptic operators by a distributed Lagrange multiplier method. C. R. Acad. Sci. Paris Sér. I Math. 327 (1998) 693-698.

[18] R. Glowinski, T.W. Pan and J. Periaux, A fictitious domain method for Dirichlet problem and applications. Comput. Methods Appl. Mech. Engrg. 111 (1994) 283-303.

[19] P. Grisvard, Singularities in boundary value problems. Springer-Verlag, Masson (1992).

[20] E. Heikkola, Y.A. Kuznetsov, P. Neittaanmäki and J. Toivanen, Fictitious domain methods for the numerical solution of two-dimensional scattering problems. J. Comput. Phys. 145 (1998) 89-109.

[21] E. Heikkola, T. Rossi and J. Toivanen, A domain embedding method for scattering problems with an absorbing boundary or a perfectly matched layer. J. Comput. Acoust. 11 (2003) 159-174.

[22] E. Hille and R.S. Phillips, Functional analysis and semigroups, Colloquium Publications 31. Rev. edn., Providence, R.I., American Mathematical Society (1957).

[23] P. Joly and L. Rhaouti. Analyse numérique - Domaines fictifs, éléments finis H(div) et condition de Neumann : le problème de la condition inf-sup. C. R. Acad. Sci. Paris Sér. I Math. 328 (1999) 1225-1230.

[24] Yu.A. Kuznetsov, Fictitious component and domain decomposition methods for the solution of eigenvalue problems, in Computing methods in applied sciences and engineering VII (Versailles, 1985), North-Holland, Amsterdam (1986) 155-172.

[25] J.C. Nédélec, A new family of mixed finite elements in $\mathbb{R}^{3}$. Numer. Math. 50 (1986) 57-81.

[26] L. Rhaouti, Domaines fictifs pour la modélisation d'un probème d'interaction fluide-structure : simulation de la timbale. Ph.D. Thesis, Paris IX, France (1999). 